\title{
Observation of Chemical Reactions in Solid Phase Using X-Ray Photoelectron Spectroscopy
}

\author{
Sergey P. Suprun, Valeriy G. Kesler and Evgeniy V. Fedosenko \\ Institute of Semiconductor Physics, \\ Siberian Branch of Russian Academy of Sciences \\ Russia
}

\section{Introduction}

Chemical reactions in solid phase are now widely used in modern technology. All electronic devices are products made on their basis. For example, such manufacturing method as vacuum epitaxy uses a sequence of chemical reactions accompanying removal of intrinsic oxides from the substrate surface and formation of heterointerface during deposition. The temperature (energy) of reagents coming from a source is as a rule much higher than the temperature of the substrate, and the atomically clean surface is highly active, all in all this creates the required reaction medium. At the next stage of layer engineering it is possible to apply electron-beam lithography, which actually is a directional spatially localized reaction in solid phase, activated by radiation, etc.

Modern engineering creates complex nanoscale objects and quantum systems with a number of specific properties, and this requires detailed knowledge about processes taking place during their formation on atomic level. One of the methods providing unique information on physical and chemical state of the surface of a solid body is a widely applied method of X-ray photoelectron spectroscopy (XPS). XPS allows us to register energy positions of deep (core) atomic levels, which is determined by the properties of external valency bonds. Change of valency bond configuration (its hybridization, length, in other words, change of the spatial distribution of charge density), which is connected with the atom environment, causes an energy shift in the underlying atomic levels (Briggs \& Seah, 1990; Sun 2007). Sensitivity of this method is high enough, so that it is possible to identify the chemical compound containing the observed element judging by the value of such shift. Since spectral measurements register electrons emitted from deep levels in the course of specimen irradiation with monochromatic X-ray radiation, then it is the electron free path in the material that determines the analysis depth. Therefore the obtained information shows the dependence between total intensity of electrons leaving the layer $\approx 1-2 \mathrm{~nm}$ thick and energy. Detailed description of the method and its characteristics can be found in a number of studies (Seah et al., 2001), so it is not going to be expounded here again. Let us consider specific examples of XPS application in development of various technologies which use the molecular beam epitaxy (MBE). 
As it was mentioned above, small analysis depth causes certain difficulties in preparation of specimens to be examined, since we need to exclude the uncontrollable chemical influence on their surface. To study the interface between two materials we need to have air lock chamber between the technological chamber for epitaxy and analysis chamber of installation in order to avoid contact with the atmosphere between operations. Another indispensable condition is a precisely controlled deposition of layers with target thickness of about $1 \mathrm{~nm}$. With such requirements met, and with the help of XPS method, it is possible to obtain unique data on heterointerface formation and structural metamorphoses on the surface (changes of crystalline state of the layer).

In order to observe changes in solid phase in situ, for example during formation of the heterointerface, it is possible to use the method described below. First, the atomically clean surface is prepared before epitaxy and then the chemical state of all elements on the surface is registered. If we acquire spectra at different angles of the specimen inclination in respect to the axis of analyzer collecting photoelectrons, we will obtain dependence of chemical state of elements on depth. It is due to the fact that effective analysis depth is defined as $d_{\mathrm{ef}}=\lambda \times \cos \theta$, where $\lambda$ is electrons path in the material, $\theta$ - is an angle between the analyser axis and the surface of the specimen. Such method makes it possible to distinguish between the surface and bulk components of the same element. Surface atoms with dangling valency bonds differ by the core levels energy position from the bulk atoms with a complete set of bonds (Sun, 2007). At the next stage a layer of another material is deposited onto the substrate at room temperature. Usually, the thickness of the layer must not exceed $1 \mathrm{~nm}$ to enable observation of chemical state of elements in the interface zone from the side of the substrate.

After deposition of the material chemical bonds can be formed even at room temperature, because of the dangled bonds existing on the surface and thermal energy of the source material. Therefore the observation of the heterointerface formation begins immediately after deposition of the layer and continues during the substrate heating. Chemical bonds in solid phase will be formed consecutively with the rise in temperature. Since the layer on the substrate surface is thin, diffusion in this case is not a limiting process. In this manner we analyzed different methods of obtaining clean surfaces of $\mathrm{Si}$, InAs, GaAs and creation of heterojunctions on their basis. This method of studying physical and chemical changes in solid phase resulted in considerable reduction of time spent to fine-tune technological modes matching optimum temperatures necessary to produce structures with required properties.

When interpreting the obtained spectra one should remember that the observed peak of an element is a sum of peaks corresponding to different states of such element within analysis depth. All this results in the change of the peak form, its widening (narrowing) with a change in chemical composition of the surface. The number of components in analysis of the observed peak is selected depending on the situation and, strictly speaking, must be justified. The process of spectral components decomposition includes operations of the energy scale shift (to compensating for the possible charging of the specimen), subtraction of the background, taking into account the shape of the peak determined by exciting radiation line width, energy resolution of the analyzer etc. Mathematical aspects of spectra processing is described in a number of specialized works. Further on we describe and discuss specific results obtained in situ using XPS method on specimens prepared according to the procedure described above. 


\section{Analysis of intrinsic InAs oxide formation in glow-discharge plasma}

Interest to metal-insulator-semiconductor structures (MIS-structures) based on $\mathrm{A}_{3} \mathrm{~B}_{5}$ compounds continuously grows owing to the high mobility of their charge carriers. However, despite the long research history, the problem of thermodynamically stable and dielectrically hard oxide formation with a low electronic states density at the interface in such semiconductors and InAs, in particular, has yet to be solved.

Direct deposition of $\mathrm{SiO}_{2}$ films on InAs surface results in the formation of a transition area with a complex chemical composition. When studying such structures, one may observe a considerable hysteresis on capacitance-voltage $(\mathrm{C}-\mathrm{V})$ characteristics, high density of surface states and pinning of Fermi level, which makes it impossible to manufacture MIS-devices on their basis (Schwartz et al., 1971; Kovchavtsev et al., 1986; Gurtov et al., 1986). Referring to the development of a new method, i.e. ALD molecular layering (Atomic Layer Deposition), research began trying to improve the parameters of MIS-structures on InAs basis, using $\mathrm{Al}_{2} \mathrm{O}_{3}$ insulator with high dielectric permittivity. The first results obtained ( $\mathrm{Li}$ et al., 2008) showed that such approach can be very promising.

So far the most widespread and the best-studied methods of formation of oxide films on the surface of $\mathrm{A}_{3} \mathrm{~B}_{5}$ semiconductors are the thermal oxidation in oxygen atmosphere, anode oxidation in liquid electrolytes and in oxygen containing plasma. The former (Leandersson et al., 2003), requires heating of the sample to high temperatures (above $350^{\circ} \mathrm{C}$ ). In the case of InAs, it causes stoichiometry disturbances in the near-surface zone of the semiconductor and in the growing oxide films because of the predominant evaporation of arsenic atoms and the processes of oxide crystallization. The ratio between As and In oxide phases in the oxide film, determining its dielectric properties, depends strongly on oxidation temperature. At higher temperature, the relative share of metal oxides increases, which results in a deterioration of the insulator electrophysical parameters. Electrophysical characteristics of MIS-structures obtained by this method are unacceptable for atomic layer engineering. Taking into account the restriction of necessarily performing all technological operations at temperatures lower than $200-250^{\circ} \mathrm{C}$, one may say that the process of thermal oxidation has no prospects.

Anode oxidation in liquid electrolytes or in oxygen-containing plasma is one of lowtemperature methods. Oxide films obtained by anode oxidation in liquid electrolytes are characterized by a diffuse interface between the insulator and semiconductor (Grigorovich et al., 1981), and by strong dependence of the ratio between As and In oxide phases and elemental arsenic on synthesis conditions (on such parameters as electrolyte acidity, anodic current density, sample temperature etc.). Moreover, there is an essential gradient of chemical compound with respect to film thickness observed (Grigorovich et al., 1981; Sorokin \& Gatko, 1985), and the structure of InAs oxides inevitably includes unwanted impurities, i.e. the electrolyte components. MIS-structures made on their basis have a number of negative properties: hysteresis phenomena, high density of surface states, unstable threshold voltage and photo-induced memory effect. The problems associated with such method of oxide layer formation are studied in great detail in a number of original works (Kornyushkin et al., 1996; Valisheva et al., 1999; Kovchavtsev et al., 2001).

There is no data in literature on InAs oxidation in plasma. From previous works (Chang et al., 1978; Ahrenkel et al., 1982) studying dry oxidation of GaAs in oxygen plasma, it follows 
that the method can be used to obtain intrinsic oxides which are homogeneous in depth and close to stoichiometry in their structure. It was found, that in the described oxidation modes with an increase of oxide thickness one may observe increase of thickness of oxide filmsubstrate transition area and increase in the amount of elemental arsenic at the interface (Chang et al., 1978). It was noted, that the accumulation of elemental As in oxide layers is accompanied by build up of the surface states. Partial improvement of electrophysical parameters of MIS-structures obtained by GaAs oxidation in oxygen plasma was observed after addition of fluorine to the oxidizing environment and after additional annealing of samples after oxidation (Ahrenkel et al., 1982). Thus, the chemical composition of oxide films on the surface of $A_{3} B_{5}$ semiconductors strongly depends on their production process, which finally determines the electrophysical properties of such MIS-structures.

Further improvement of the intrinsic oxide quality on the surface of binary $\mathrm{A}_{3} \mathrm{~B}_{5}$ type semiconductors is possible using low-temperature oxidations in plasma. Such process can be performed at room temperature, in a vacuum chamber, using high-purity oxidizing gases. It offers a great advantage compared with thermal and anode oxidation methods, making it possible to exclude the effects related to stoichiometry disturbance and contamination of the oxide.

In the next section we describe the results of our study of chemical composition and formation kinetics of super-thin (5-10) nm intrinsic oxide films on the surface of InAs in oxygen plasma, in normal and glow discharge plasma, and the effects of cathode and anode sample polarization and influence of gas atmosphere, obtained in situ using the XPS method.

\subsection{Preliminary sample preparation}

Formation of super-thin oxide layers imposes special requirements for purity and surface relief of the initial samples. Chemical finishing of the surface of InAs plates was carried out using the technique described by (Kesler et al., 2010). Before loading into a vacuum chamber the samples were degreased by boiling in toluene for 10 minutes, then treated with a saturated solution of hydrochloric acid in isopropanol (HCl-IPA) for 5-30 minutes at room temperature. Immediately after chemical processing and rinsing in isopropanol, the samples were loaded into ultrahigh-vacuum chamber with working pressure less than $1 \times 10^{-9}$ Torr.

Chemical composition and oxide films thickness were studied using the XPS method. For XPS spectra excitation we used a non-monochromatic aluminium source with line energy $\mathrm{Al}$ $\mathrm{K}_{\alpha}$ of $1486.6 \mathrm{eV}$. All XPS-spectra were acquired with analyser resolution $\Delta \mathrm{E}=0.7 \mathrm{eV}$, which corresponds to $\mathrm{Au} 4 \mathrm{f}_{7 / 2}$ line width at half-height equalling $1.31 \mathrm{eV}$. The device was calibrated using $\mathrm{Cu} 2 \mathrm{p}_{3 / 2}, \mathrm{Cu} 3 \mathrm{p}_{3 / 2}$ and $\mathrm{Au} 4 \mathrm{f}_{7 / 2}$ lines, which ensured precision of the energy position registration not less than $0.1 \mathrm{eV}$. Signal electrons were registered at the angle of $20^{\circ}$ off the normal to the sample surface. Overlapping photoelectron peaks were resolved into separate components (Gauss and Lorentz functions) using XPSPEAK95 Version 2.0 software. The parameters of the form of photoelectronic lines were determined through measurement of freshly made chemically pure standard reference samples: InAs and anode oxide films on InAs surface formed in the alkaline electrolyte.

XPS-spectra of the samples surface at different stages of their finishing cleaning are shown in Fig. 1. After degreasing in toluene there is an oxide film of the surface of samples about 2 
$\mathrm{nm}$ thick consisting of In and As oxides. Etching in HCl-IPA solution for 5 minutes results in almost complete removal of oxides, but some traces of hydrocarbon impurities, oxygen and chlorine, still remain on the surface. Prolonged treatment does not lead to substantial reduction of residual impurities concentration and, at the same time, monotonous increase in the concentration of elemental arsenic on the surface of samples (Fig. 2) is observed.

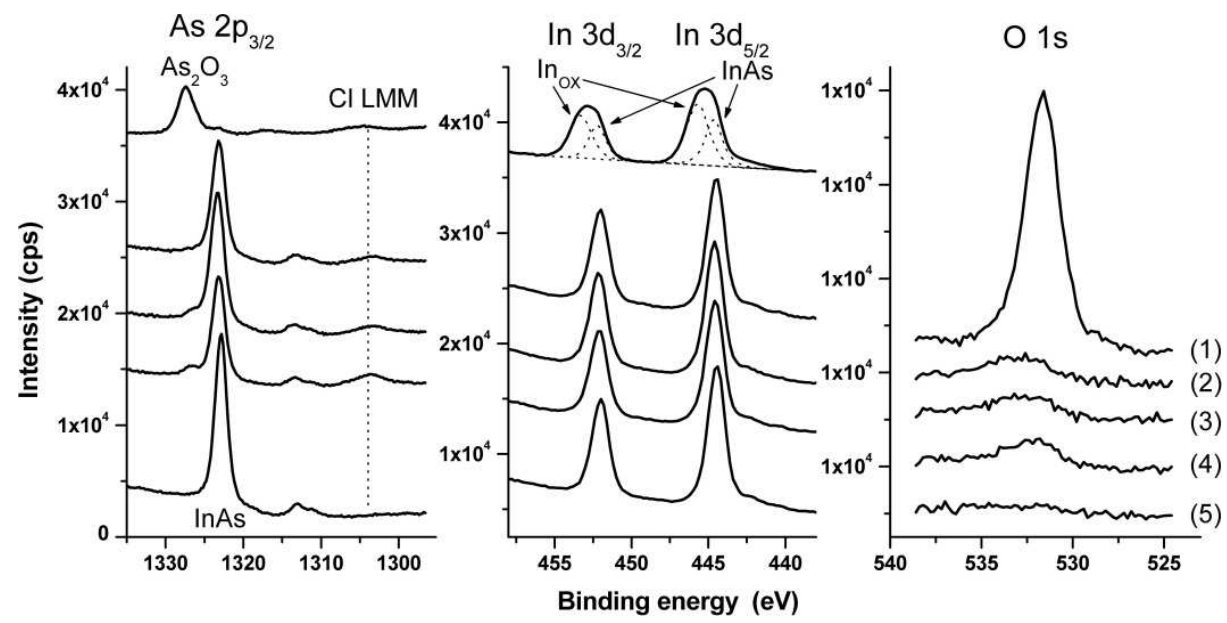

Fig. 1. XPS lines of As, In, and O on the surface of InAs (111)A sample: (1) - after degreasing in toluene, (2), (3), and (4) - after HCl-IPA treatment for 5, 15, and 30 minutes, respectively, (5) - after 60 -min of vacuum annealing at $300^{\circ} \mathrm{C}$.

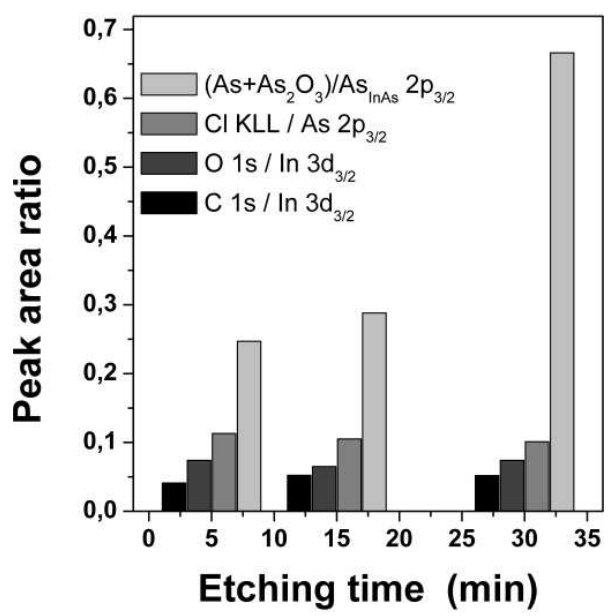

Fig. 2. Peak area ratios observed in the samples treated in isopropanol saturated with hydrochloric acid as a function of etching time.

Such processing results in surface passivation, practically not changing its average roughness value, and also increases lifetime of nonequilibrium charge carriers in the near- 
surface zone of the samples. However, in this case we may observe formation of local microdefects in the form of "hillocks" consisting mainly of arsenic and its oxides a few nanometers high and several tens of nanometers wide, and with surface density up to $3 \times 10^{8}$ $\mathrm{cm}^{-2}$ (Kesler et al., 2010). Chemical reactions which can supply excessive arsenic for formation of such "hillocks" are discussed in (Sun et al., 2008).

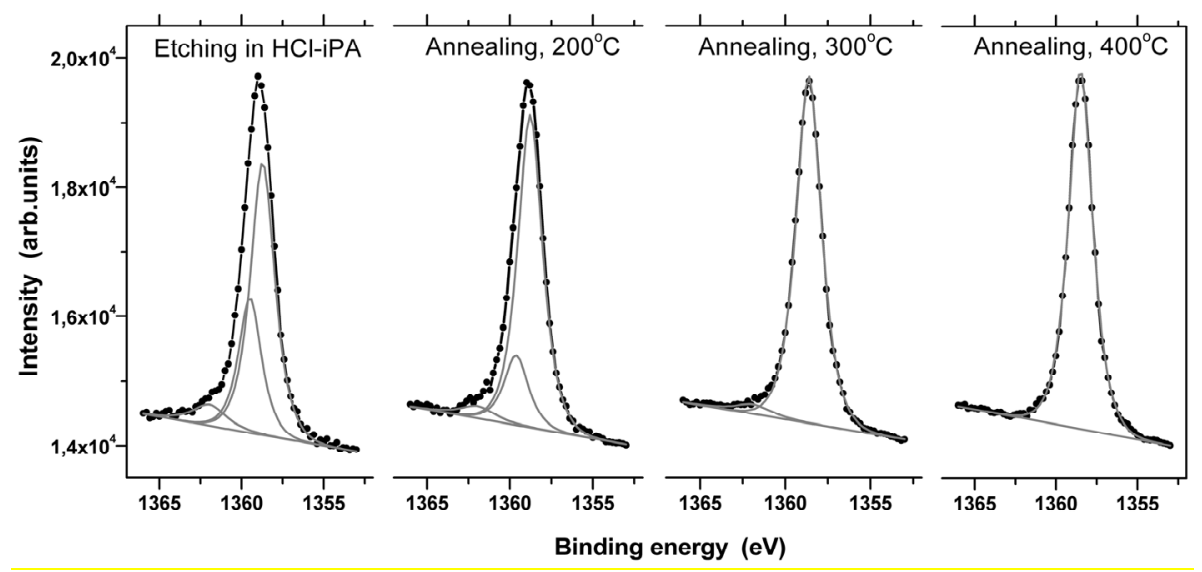

Fig. 3. Detailed spectra of As $2 \mathrm{p}_{1 / 2}$ and deconvolution results for the surface of InAs after etching in $\mathrm{HCl}-\mathrm{IPA}$ solution and vacuum annealing.

Finishing cleaning of the surface of InAs samples (111)A was performed using vacuum annealing. XPS method in situ is literally irreplaceable when it comes to monitoring purity of the surface. Since photoelectrons escape depth for As $2 \mathrm{p}_{1 / 2}$ line is $\approx 0.6 \mathrm{~nm}$, this peak is the most sensitive to the changes in the surface chemical composition. Fig. 3 shows the peak and its decomposition into three components. Main component with binding energy of $1358.7 \mathrm{eV}$ is related to arsenic atoms in InAs volume, and components with energies of $1359.5 \mathrm{eV}$ and $1362.0 \mathrm{eV}$ are related to elemental As and its oxide in the surface layer. The ratio between intensities of the surface and bulk peak components allows us to calculate the thickness of the covering surface layer (Tereshchenko et al., 2006). After the sample is etched in HCl-IPA solution for 30 minutes thickness of the covering arsenic layer is $0.2 \mathrm{~nm}$, which corresponds to 1 monolayer (ML). After 30 minutes of vacuum annealing at $200^{\circ} \mathrm{C}$ the degree of surface coating with elemental arsenic decreases approximately by $50 \%$, and annealing at $300^{\circ} \mathrm{C}$ results in complete absorption of excessive As. Complete removal of As oxides and $\mathrm{Cl}$ traces is observed at temperatures about $400^{\circ} \mathrm{C}$. Hydrocarbon impurities are not completely removed even at a temperature $\sim 500^{\circ} \mathrm{C}$. Minimal value of carbon peak is 30 $\%$ from its initial value, which corresponds to surface coating with carbon $\sim 0.1 \mathrm{ML}$.

Comparison of intensities of As $3 \mathrm{~d}$ and In $4 \mathrm{~d}$ photoelectronic peaks characterized by similar photoelectrons escape depth, allows us to estimate changes in stoichiometry of the nearsurface zones of the samples. The results of the composition measurements at InAs surface during various stages of vacuum annealing are shown in Fig. 4. Increase in the annealing temperature up to $(200-500){ }^{\circ} \mathrm{C}$ leads to the monotonous decrease of relative content of As and at the same time to a decrease in lifetime of minority charge carriers according to the results of electrophysical measurements. 
It is widely thought that the first thing that takes place during the low-temperature annealing of InAs is evaporation of excessive elemental arsenic accumulated the surface after etching in HCl-IPA. On the other hand, at higher temperatures As is evaporated from the substrate. Vacuum annealing does not result in any significant changes in average surface roughness, but at temperatures exceeding $300^{\circ} \mathrm{C}$ one may observe complete disappearance of such surface microdefects as "hillocks" (Kesler et al., 2010). Evaporation of elemental arsenic decreases the effects of surface passivation. Similar regularities were found in (Dultsev \& Kesler, 2009); the results of studies related to accumulation of As at InAs surface may be found in (Sun et al., 2008; Tereshchenko et al., 2003).

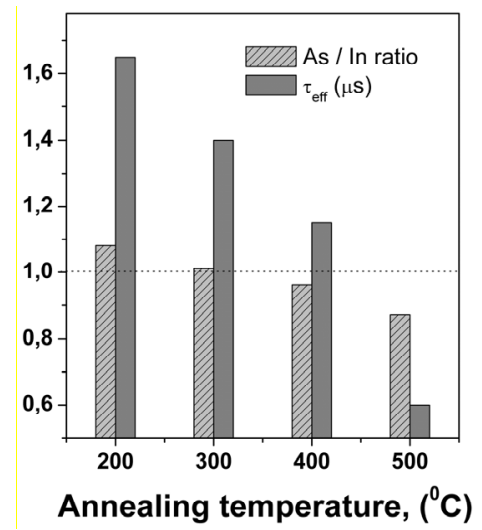

Fig. 4. As / In surface concentration ratio and effective lifetime of minority charge carriers in the subsurface zone of InAs samples vacuum-annealed in the temperature range $(200-500)^{\circ} \mathrm{C}$.

Optimal preparation modes of the surface of InAs (111) are surface etching in HCl-IPA solution for 5-30 minutes and subsequent annealing of samples in vacuum for 30-60 minutes at temperatures about $(300-350)^{\circ} \mathrm{C}$. Higher annealing temperatures cause significant disturbance of surface stoichiometry due to predominant evaporation of As, which leads to the reduction of effective life time of non-equilibrium charge carriers. This method of preliminary preparation ensures high purity, stability and inertness of InAs surface.

\subsection{Results of studying InAs oxidation in glow-discharge plasma}

Oxidation process was conducted directly in one of high-vacuum modules of the surface analysis installation, which allowed us to control the sample chemical composition immediately after each cycle of plasma processing without any contact with the atmosphere. The examined samples were mounted on a molybdenum holder enabling electric contact to the aluminium auxiliary electrode put under positive/negative electric potential in relation to the frame of the vacuum chamber. During our experiments the sample was put in a stable glow discharge column, which ensured uniformity of surface processing. The experimental procedure is described in detail in (Kesler et al., 2010a; Kesler, 2011).

Total thickness of the films being grown was determined using XPS method by a component of As $3 \mathrm{~d}$ peak with binding energy of $41.1 \mathrm{eV}$ (InAs substrate) assuming exponential reduction of signal intensity with the increase of oxide thickness. In order to estimate the 
thickness of the film of InAs intrinsic oxide, we used the approach developed in (Seah \& Spenser, 2002) based on a measurement of the relation between integral intensities of photoelectronic peaks of oxide films and the substrate:

$$
\mathrm{d}=\lambda_{\mathrm{ox}} \cdot \cos (\theta) \cdot \ln \left(1+\frac{\mathrm{I}_{\mathrm{sub}}^{\infty} \cdot \mathrm{I}_{\mathrm{ox}}}{\mathrm{I}_{\mathrm{ox}}^{\infty} \cdot \mathrm{I}_{\mathrm{sub}}}\right)
$$

where $d$ is the thickness of the oxide film, $I_{\text {sub }}^{\infty}$ and $I_{o x}^{\infty}$ - integral intensities of As $3 d$ peaks from semi-infinite substrate samples and anode oxide film measured in similar experimental conditions, $I_{\mathrm{ox}}$ and $I_{\mathrm{sub}}$ - measured integral intensities of As $3 \mathrm{~d}$ peak components with binding energy $\sim 45 \mathrm{eV}$ (oxide film) and $\sim 41 \mathrm{eV}$ (substrate), $\lambda_{\text {ox }}$ - (attenuation length) of non-elastic dispersion of $3 \mathrm{~d}$ As electrons in the film, $\theta$ - registered electrons emission angle off the normal to the sample surface. In our calculations we used $\lambda_{\mathrm{ox}}=3.08 \mathrm{~nm}$.

When studying basic regularities and features of InAs oxidation in glow discharge plasma, we analyzed three modes:

1 - the sample was under negative potential in relation to the auxiliary electrode, and voltage in the spark gap was close to glow discharge ignition voltage;

2, 3 - the sample was under positive potential in relation to the auxiliary electrode, and voltage in the spark gap corresponded to the dark and normal glowing discharge, respectively.

Choice of the range from the dark discharge to the beginning of plasma glow is related to the fact that the smallest radiation disturbances of the surface of studied sample are observed in this range. Moreover, plasma of electronegative gases $\left(\mathrm{O}_{2}, \mathrm{CO}_{2}\right)$ must show the effect of "sticking" electrons with formation of negative oxygen ions necessary for oxidation of the sample located on the anode.

Using cathode polarization (negative potential on the sample), the sample surface is bombarded by positive oxygen ions with energies determined by a potential difference in the spark gap. Formation of oxide films is accompanied by oxygen ions penetrating deep inside the sample and formation of chemical bonds with the lattice atoms. Fig. $5 a$ shows XPS lines of arsenic and indium for InAs (111)A sample after chemical preparation and stage-bystage processing in oxygen plasma for 10 - 50 minutes.

The surface of the samples is not oxidized in oxygen atmosphere immediately after chemical preparation at pressure of 0.15 torr for a long exposure time (more than 65 minutes) (Kesler et al., 2010a). Binding energy and full width at half-height (FWHM) of As 3d and In 4d peaks is respectively 41.1 (1.7) $\mathrm{eV}$ and 17.7 (1.8) eV. After ignition of the glow discharge plasma an additional peak with binding energy $45.4 \mathrm{eV}$ and width $\approx 2.3 \mathrm{eV}$ appears in As $3 \mathrm{~d}$ spectrum, corresponding to the oxide state of As atoms. Analysis of the peak form shows that it is a superposition of two components with binding energies $45.7 \mathrm{eV}$ and $44.4 \mathrm{eV}$, identified as states $\mathrm{As}^{5}{ }^{+}$and $\mathrm{As}^{3+}$. At the same time one may observe a shift of In $4 \mathrm{~d}$ line toward higher binding energies up to $18.2 \mathrm{eV}$ and its widening up to $2.4 \mathrm{eV}$, indicating appearance of indium oxides. Obtained experimental peaks In $4 \mathrm{~d}$ can be well described by superposition of just two components with binding energies - $17.7 \mathrm{eV}$ (InAs) and $18.8 \mathrm{eV}$ (indium oxide). Results of our analysis of oxidation kinetics and chemical composition of 
near-surface zone of the sample during various oxidation stages are shown in Fig. $5 b$. It can be seen, that values of thickness of oxide films on the surfaces calculated from the attenuation of As 3d (substrate) peak and from the ratio between oxidized and non-oxidized arsenic components, are quite consistent. In this case the thickness of oxide films does not really depend on oxidation time and is about $2.6 \mathrm{~nm}$.

a

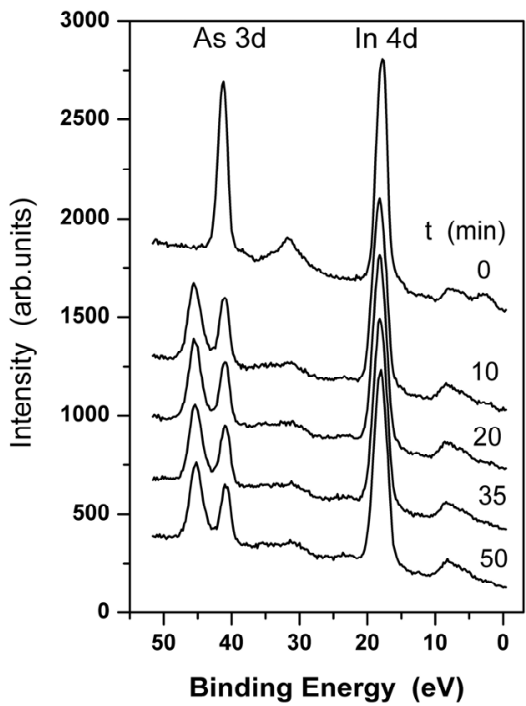

b

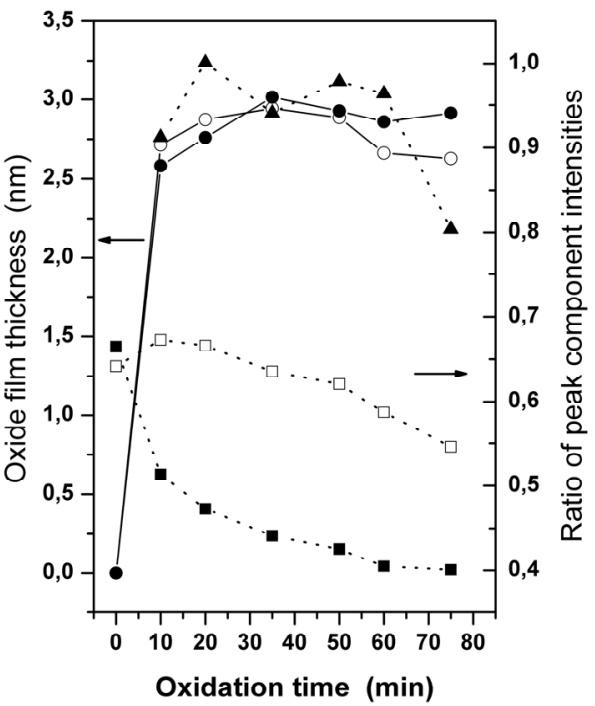

Fig. 5. XPS spectra of As 3d and In 4d in InAs sample during various stages of its processing in oxygen glow discharge plasma at sample voltage of $350 \mathrm{~V}(\mathrm{a})$, experimental values of the relation between integrated intensities of peak components and calculated values of thickness of the formed oxide films (b): ( $\square$ ) - total relation of As 3d / In 4d peak components intensities; ( $\mathbf{-}$ - relation of peak components intensities of As 3d / In $4 \mathrm{~d}$ connected with the substrate; $(\boldsymbol{\Delta})$ - relation of peak components intensities of As $3 \mathrm{~d} / \mathrm{In} 4 \mathrm{~d}$ connected with the oxide film; $(\bullet)$ - calculation of the total thickness of oxide films from the absolute attenuation of As 3d peak from the substrate; (०) - calculation of the thickness of oxide films from the ratio of As $3 \mathrm{~d}$ peak components connected with the oxide film and InAs substrate.

Increase in oxidation time results in observed monotonous reduction of the relation between integral intensities of As $3 \mathrm{~d}$ and In $4 \mathrm{~d}$ peaks, but the relation between oxide components of those peaks changes only slightly. Most significant reduction in relative arsenic content in relation to indium in the near-surface zone is observed near the boundary between the substrate and the oxide film.

The results of our study of InAs oxidation kinetics when the anode polarization is used (positive potential on the sample) are shown in Fig. 6. In this case the negatively charged oxygen ions take part in oxidation. In the dark discharge mode there is no visible discharge glow, the potential difference in the spark gap is $320 \mathrm{~V}$ and it is close in its absolute value to the cathode polarization mode described above. In the normal glow discharge mode the potential difference is $500 \mathrm{~V}$, and bright yellow glow is observed. 
After discharge ignition, an oxide film begins to form on the surface just as in case of cathode polarization, as evidenced by a change in the acquired XPS spectra for indium and arsenic (Fig. $6 \boldsymbol{a}, \boldsymbol{b}$ ). Peculiarities of the changes in shape and position of As $3 \mathrm{~d}$ and In $4 \mathrm{~d}$ lines, observed earlier, are qualitatively repeated. Most evident distinctions are observed in the oxidation process kinetics (Fig. $6 b, d$ ). In anode polarization mode, with the increase of processing time in plasma, we observe monotonous increase in the total thickness of the film, calculated from the attenuation of As 3d (substrate) peak. Estimations of thickness of intrinsic oxide films on the surface of InAs samples, made by the ratio of oxidized and not oxidized As components, give smaller values. The difference between total thickness of oxide films and thickness of intrinsic InAs oxide grows linearly at the rate of $0.001 \mathrm{~nm} \times \mathrm{min}^{-1}$ and $0.03 \mathrm{~nm} \times \mathrm{min}^{-1}$ with time of processing in plasma for discharge voltage $+320 \mathrm{~V}$ and +500 $\mathrm{V}$, respectively. Growth of intrinsic oxide on InAs films in dark discharge proceeds logarithmically with time $(d[\mathrm{~nm}]=0.38+0.3 \ln (t))$, growth of intrinsic oxide film in the glow discharge mode occurs only at initial oxidation stages, then the saturation is attained and an insignificant reduction of oxide thickness is observed. In the glow discharge mode $(+320 \mathrm{~V})$ the relation between integral intensities of $\mathrm{As} 3 \mathrm{~d}$ and In $4 \mathrm{~d}$ peaks and intensities of components connected with the film and the substrate, does not really depend on duration of plasma processing.

Glow discharge mode $(+500 \mathrm{~V})$ shows strong dependence between the relation of integral intensities of As $3 \mathrm{~d}$ and In $4 \mathrm{~d}$ peaks and their components on oxidation process duration. At the initial stages of InAs oxidation, considerable growth of oxide arsenic component in relation to similar indium component is observed: the relation between $\mathrm{As}_{\mathrm{ox}} / \mathrm{In}_{\mathrm{ox}}$ components reaches 2.5. At the same time one may observe a reduction of relative arsenic content in relation to indium in the zone near the substrate - oxide film interface.

Relative share of $\mathrm{As}^{5}+$ in oxide goes down during later oxidation stages (Fig. $6 a$ ), and the ratio $\mathrm{As}_{\mathrm{ox}} / \mathrm{In}_{\mathrm{ox}}$ decreases to 1.0. With the increase in InAs processing duration in plasma for over 50 minutes, one may observe the growth of the ratio between arsenic and indium components, related to the substrate, and the increase in As $3 \mathrm{~d}$ peak width up to $\approx 3 \mathrm{eV}$, which indicates appearance of arsenic atoms in the oxide film with lower oxidation degree and/or lower content of elemental arsenic. Considerable intensity reduction of this component of As $3 \mathrm{~d}$ peak with the increase in oxide film thickness in the course of oxidation makes more exact estimations difficult.

Fig. 7 shows XPS spectra of As, In and Al for the samples with an oxide film synthesized using various gases in different plasma modes. Films obtained in oxygen plasma consist of a mixture of indium and arsenic oxides. The contribution of arsenic oxides in them exceeds the contribution of indium oxides, and the contribution of $\mathrm{As}^{5+}$ oxide exceeds that of $\mathrm{As}^{3+}$ oxide. Addition of $\mathrm{NF}_{3}$ gas to oxygen plasma leads to a substantial growth of oxidation rate (up to 35 times in case of $100 \%$ content of $\mathrm{NF}_{3}$ ) and to a strong shift of chemical composition towards formation of indium and arsenic fluorides. All indium in the film is combined with fluorine, and arsenic peak represents superposition of several arsenic oxyfluorides. The composition of the formed film also includes an insignificant amount of nitrogen.

Presence of aluminium peaks in XPS spectra indicates that the material is transferred from the cathode to the anode with the simultaneous oxidation. It explains observed distinctions between the obtained estimates of the total thickness of oxide films and the thickness of 
InAs intrinsic oxide (Fig. $6 b, d$ ). Growing film of aluminium oxide acts as a barrier preventing further oxidation during the diffusion of oxygen to the surface of InAs. Deposition rate of aluminium oxide film is strongly suppressed if low discharge voltage is used, and with the increase in gas pressure. In these cases no fast formation of a continuous barrier layer of aluminium oxide is observed, and aluminium is built into the growing oxide film of InAs. This given assumption is confirmed by chemical shift of $\mathrm{Al} 2 \mathrm{p}$ peak (Fig. 7) which depends on the synthesis conditions.

a

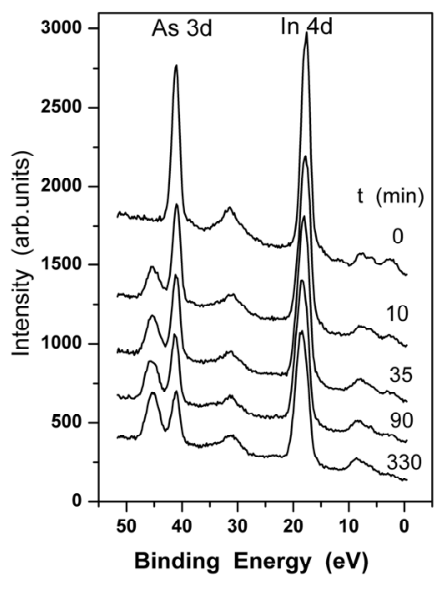

c

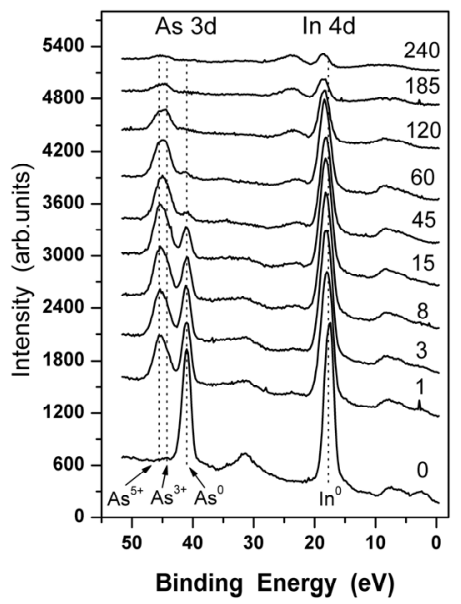

b

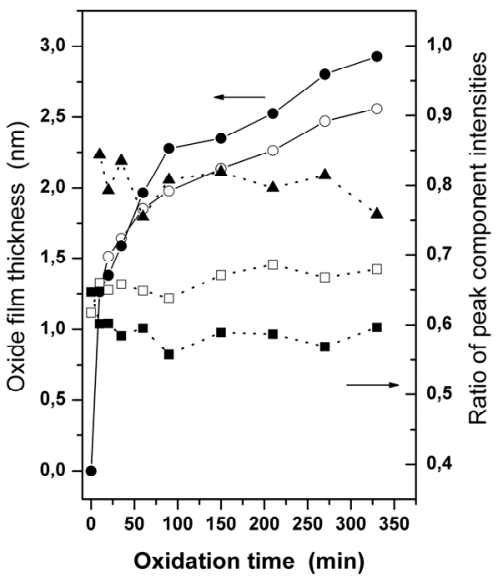

d

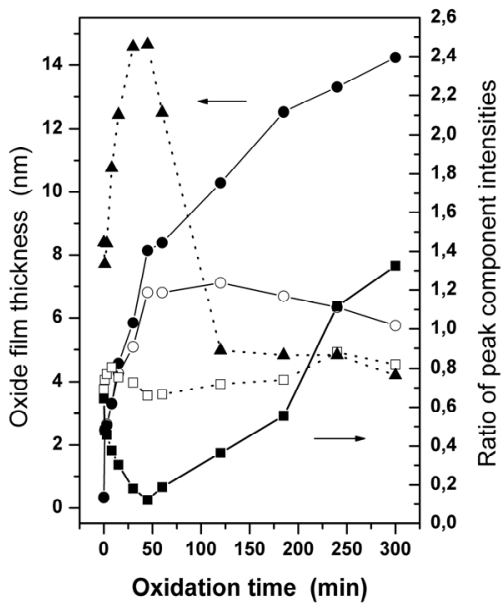

Fig. 6. X-ray photoelectron spectra of As $3 \mathrm{~d}$ and In $4 \mathrm{~d}$ from the surface of InAs sample at various oxidation stages in oxygen glow discharge plasma $\left(\mathrm{NF}_{3}\right.$ gas content is less than $1 \%$ ) at sample voltage $+320 \mathrm{~V}(\boldsymbol{a})$ and $+500 \mathrm{~V}(\boldsymbol{c})$. Experimental values of the ratio between integral intensities of peak components and calculated values of oxide film thickness being formed at sample voltage $+320 \mathrm{~V}(\boldsymbol{b})$ and $+500 \mathrm{~V}(\boldsymbol{d})$. The designations correspond to those used in Fig. 5. 

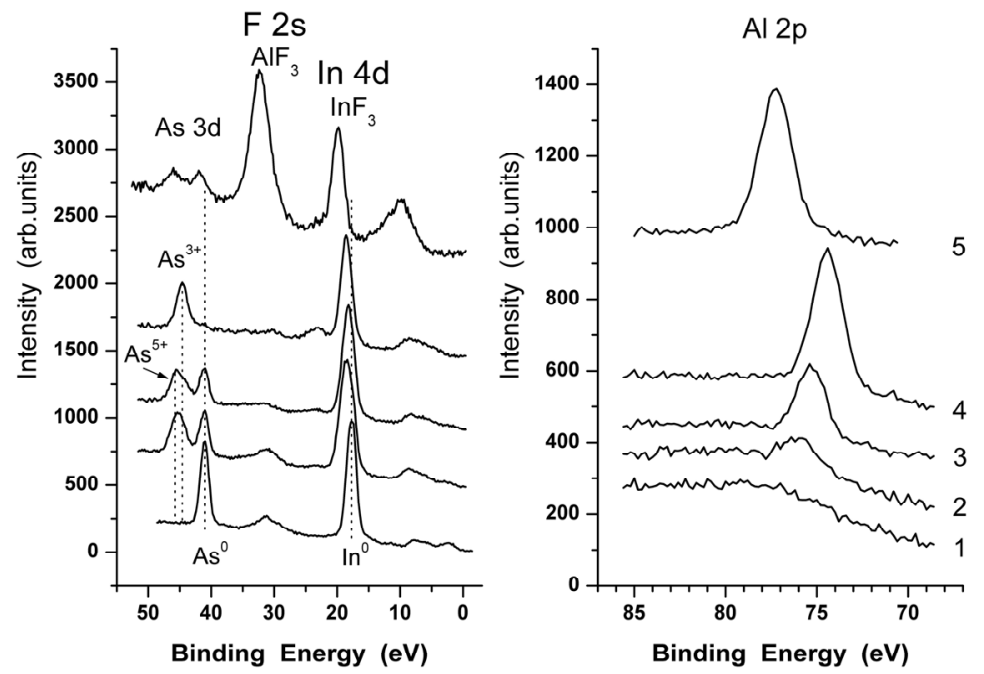

Fig. 7. X-ray photoelectron spectra from the surface of InAs samples: (1) -initial sample after chemical cleaning; (2) - after oxidation in plasma $\left(\mathrm{O}_{2}+\right.$ less than $1 \%$ NF3) for 330 minutes, at pressure 0.15 Torr, voltage $+320 \mathrm{~V}$, current $0.17 \mathrm{~mA}$; (3) - after oxidation in $\mathrm{O}_{2}$ plasma (no $\mathrm{NF}_{3}$ gas in plasma) for 65 minutes, at pressure 0.15 Torr, voltage $+500 \mathrm{~V}$, current $1.5 \mathrm{~mA}$; (4) - after oxidation in plasma $\left(\mathrm{CO}_{2}+\right.$ less than $\left.1 \% \mathrm{NF}_{3}\right)$ for 2220 minutes, at pressure 0.5 Torr, voltage $+375 \mathrm{~V}$, current $1.0 \mathrm{~mA}$; (5) - after oxidation in plasma $\left(\mathrm{O}_{2}+7 \% \mathrm{NF}_{3}\right)$ for 65 minutes at pressure 0.15 Torr, voltage $+500 \mathrm{~V}$, current $1.5 \mathrm{~mA}$.

The information on chemical composition and kinetics of oxide films growth on InAs surface, obtained for the first time in situ with the help of XPS method, made it possible to find out the following features of oxide films growth in glow discharge plasma using $\mathrm{O}_{2}$, $\mathrm{CO}_{2}$ gases and gas mixtures $\mathrm{O}_{2}$ and $\mathrm{NF}_{3}$ :

1. An oxide film several nanometers thick is formed on the sample surface during cathode polarization as a result of sample bombardment by positive ions of oxygen plasma. Thickness of the oxide film does not really change with increase in duration of surface processing, but the ratio between As/In concentrations both in oxide film and in nearsurface zone of the semiconductor decreases. By varying the discharge voltage, it is possible to control the oxide thickness, which indicates the dominating role of ion sputtering in the process.

2. Two-layer oxide films are formed on the surface of InAs during the anode polarization of the sample in a glow discharge mode. The bottom layer, several nanometers thick consisting of arsenic and indium oxides, is formed at the initial stages of oxidation, and later its thickness does not practically change. The top layer - $\mathrm{Al}_{2} \mathrm{O}_{3}$ serves as a barrier to oxygen diffusion, and its thickness grows linearly with the time of processing in glow discharge plasma. The chemical composition of the growing intrinsic InAs oxide film and the stoichiometry of the near-surface substrate zones depend strongly on the parameters of oxidation process. Voltage reduction in the spark gap and/or increase in gas pressure result in decrease in the growth rate of InAs oxide films, and lead to embedding of cathode material atoms in their oxide form into the film. 
3. Use of $\mathrm{O}_{2}+7 \% \mathrm{NF}_{3}$ gas mixture or $100 \%$ of $\mathrm{NF}_{3}$ gas causes sharp increase in oxide film growth rate -7 and 35 times, respectively. Fluorine causes attenuation of barrier properties of the aluminium oxide protective layer. Indium and arsenic are present in top layers of oxide films even if they are 35-85 nanometers thick.

4. In films generated in oxygen plasma, the contribution of arsenic oxides exceeds that of indium oxides. Addition of $\mathrm{NF}_{3}$ gas to oxygen plasma results in a strong shift in synthesis towards formation of indium and arsenic fluorides. All indium in the film is combined with fluorine, and the arsenic peak is a superposition of several components -arsenic oxyfluorides. Composition of the generated film also includes nitrogen.

5. Mostly arsenic oxides $\mathrm{As}_{2} \mathrm{O}_{5}$ are formed at the initial stages of formation of InAs oxide films in $\mathrm{O}_{2}$ and $\mathrm{CO}_{2}$ plasma. With a rise in the film thickness, the share of five-valency arsenic oxide decreases, and the share of trivalent arsenic oxide grows.

The conducted research shows the possibility of controllable and precise growth of tunnelthin oxide films to be used in various microelectronic and nanoelectronic devices. The results described in (Guzev et al., 2008; Kesler et al., 2010a; Kesler et al., 2011) showed that it is probably possible to passivate the surface of InAs with oxide films 3-5 nm thick formed in glow discharge plasma, which opens new prospects for modern device engineering.

\section{Formation of platinum silicide on silicon}

There are a number of problems in modern production technology of photodetector arrays, sensitive in spectral range not typical for silicon, the primarily used semiconductor material. The systems for signals processing are made of elements mounted on a silicon wafer, and the photodetectors are the areas of other material connected to the substrate in a defined way. Photosensitive elements are sometimes grown in special remaining windows on a wafer, with the electronic circuit already formed on it. And elements need to be formed on an atomically clean substrate surface. It is well known that the intrinsic silicon oxide is removed from the surface only at high temperature, which may deteriorate the properties of the electronic part of the array. One example of similar technology is creation of photodetector arrays on $p$-Si-PtSi Schottky barriers, sensitive in 5-micron range.

The following chemical way of $\mathrm{Si}$ processing was suggested, in order to reduce the temperature necessary to obtain atomically clean surface in such windows. The first stage includes operations performed according to the standard RCA technique. The next step is a finishing etching of the rotating wafer, using a solution of fluoric acid in ethanol in the ratio 1:20 in inert gas atmosphere in a hermetic box (Grunthaner et al., 1989). This operation allows "deposition" of hydrogen on dangling silicon bonds on the surface, and the surface is hydrogenised. Then the wafer is loaded into a hermetic container and put into the load lock of the vacuum installation where inert gas is pumped out, and the specimen is transferred to the MBE growth chamber. Presence of hydrogen stops the reaction of silicon oxidation and prevents oxide formation. When such substrate is heated in high vacuum at temperatures about $300^{\circ} \mathrm{C}$, Si-H bonds get broken exposing the atomically clean surface ready for further operations.

However, the process of Schottky barrier formation using the above-described way of cleaning, showed strong dependence of electrophysical properties on the time interval between the last operation of chemical treatment and the vacuum processing of the 
specimen (Grunthaner et al., 1989). Analysis of hydrogenised Si surface showed that Auger and XPS spectra contain peaks corresponding to carbon and oxygen after exposure of the wafer to the atmosphere, but the chemical state of silicon corresponds to a clean material. This means that the presence of $\mathrm{Si}-\mathrm{H}$ bonds on silicon surface protects it from oxidation, and the registered elements are in a physically adsorbed state. Nevertheless, some aspects of such protection are still unclear, such as the necessity of inert gas after processing, dependence on time of exposure to the atmosphere, etc.

To better understand the mechanism behind this process, we set up the following experiment in our laboratories (Suprun et al., 1995). After hydrogenization of the surface, the specimen was exposed to water vapours, and then loaded into the analysis chamber. With $\mathrm{Mg} \mathrm{K} \mathrm{K}_{\alpha}$ line used as the source of radiation, with energy $1253.6 \mathrm{eV}$, XPS spectrum of the initial surface featured peaks corresponding to oxygen and unoxidized silicon. Thus the hydrogen passivation of dangling silicon bonds renders its protective effect on surface. In the subsequent heating of the specimen in analysis chamber Si $2 p$ peak was recorded (Fig. 8). No significant changes in the spectrum were observed up to the temperature about $350^{\circ} \mathrm{C}$, and the further heating resulted in oxidation of silicon surface. Fig. 8 shows the beginning and the end at $450^{\circ} \mathrm{C}$ of the process of $\mathrm{Si} 2 \mathrm{p}$ "flow" from the clean to oxidized state. One may conclude that the desorption energy increases on such "van der Waals" surface stabilized by hydrogen. As a result of specimen heating, the breakdown of Si-H bonds occurs earlier than the evaporation of physically adsorbed water, which leads to oxidation of silicon. It means that in order to obtain clean surface in such a way, it is necessary to exclude the physical adsorption of compounds leading to its oxidation. Moreover, it is recommended to reduce water content in initial reagents as much as possible, for example, by using dehydrated ethanol.

Sensitive elements of photodetectors array on a basis of Schottky barrier $p$-Si-PtSi are formed by deposition of a layer of platinum $2-3 \mathrm{~nm}$ thick in the prepared windows with subsequent heating of the wafer in high vacuum. Fine-tuning of this technology requires detailed knowledge of the processes taking place during possible formation of platinum silicides - $\mathrm{Pt}_{2} \mathrm{Si}$ and $\mathrm{PtSi}$. For this reason we developed a model experiment on silicon substrate, to observe changes in the chemical state of Si-Pt system with heating. Here we need to take into account some features of the XPS method. Photoelectrons during the spectrum acquisition are picked up from an area about $0.5 \mathrm{~cm}$ in diameter, and the signal is therefore integrated over that area. This imposes strict requirements over the uniformity of thickness of the deposited layer in the area to reduce additional widening of registered peaks.

To monitor the thickness of nanoscale coatings on monocrystal substrate we used Raman scattering method (RS), registering the spectrum of Raman emission of the substrate (Suprun et al., 1995a). Intensity of the emission passing through the deposited layer exponentially falls with its thickness, allowing us to determine its characteristics. The atomic resolution electron microscopy of the structure cross-sections was used for independent calibration.

According to the procedures described above, a Pt layer was deposited on a clean Si surface at room temperature and then the specimen was heated and XPS spectra was acquired. Obtained $\mathrm{Pt} 4 \mathrm{f}_{7 / 2}$ peaks were approximated with Gauss functions and their area, position 
and width were determined. Such spectrum analysis methodology is rather qualitative, since it does not imply precise decomposition of peaks into components, but nevertheless it is fairly informative.

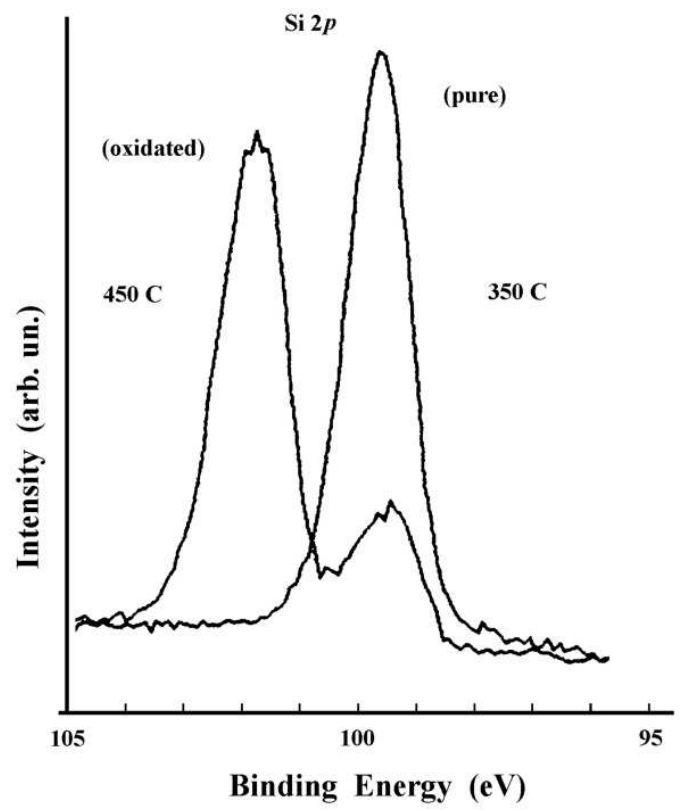

Fig. 8. Changes in XPS spectra of the sample surface during annealing at $450^{\circ} \mathrm{C}$.

The inset in fig. 9 shows the evolution of $\mathrm{Pt} 4 \mathrm{f}$ peaks for a specimen with a layer $15.6 \mathrm{~nm}$ thick during its heating. It is clearly seen that the spectrum with time (each spectrum acquisition took 6 minutes) is shifting towards lower kinetic energy, which corresponds to an increase in electron binding energy in atom, and peaks intensity is decreasing as well. The figure shows the results of $\mathrm{Pt} 4 \mathrm{f}_{7 / 2}$ peak processing by the position of its maximum and by its width at half-height. The position of the maximum (black circles, right curve) shifts by more than $1 \mathrm{eV}$. Peak width here (open circles, left curve) is actually a time derivative of the first curve. The obtained dependences reflect the change of Pt chemical state within the analysis depth, transition of the pure metal into silicide, and the peak widening due to registration of element in two different states.

Fig. 10 shows the behaviour of the area under peaks in the spectra of two specimens with average metal thickness of 4.5 and $15.6 \mathrm{~nm}$ during annealing: the first one was annealed at $300^{\circ} \mathrm{C}$ and the second at $400^{\circ} \mathrm{C}$. It can be seen that platinum concentration on the surface falls by half during heating, asymptotically reaching $50 \pm 5 \%$, which presumably corresponds to the formation of PtSi compound. These curves have different shape determined by the course of reaction in films of different thickness. Reaction at the interface, as it was demonstrated on layers less than $3 \mathrm{~nm}$ thick, begins with $\mathrm{Pt}$ deposition on the specimen at room temperature, owing to high temperature of the source - the electron beam evaporator (about $2000^{\circ} \mathrm{C}$ ) and exothermic nature of the reaction. 


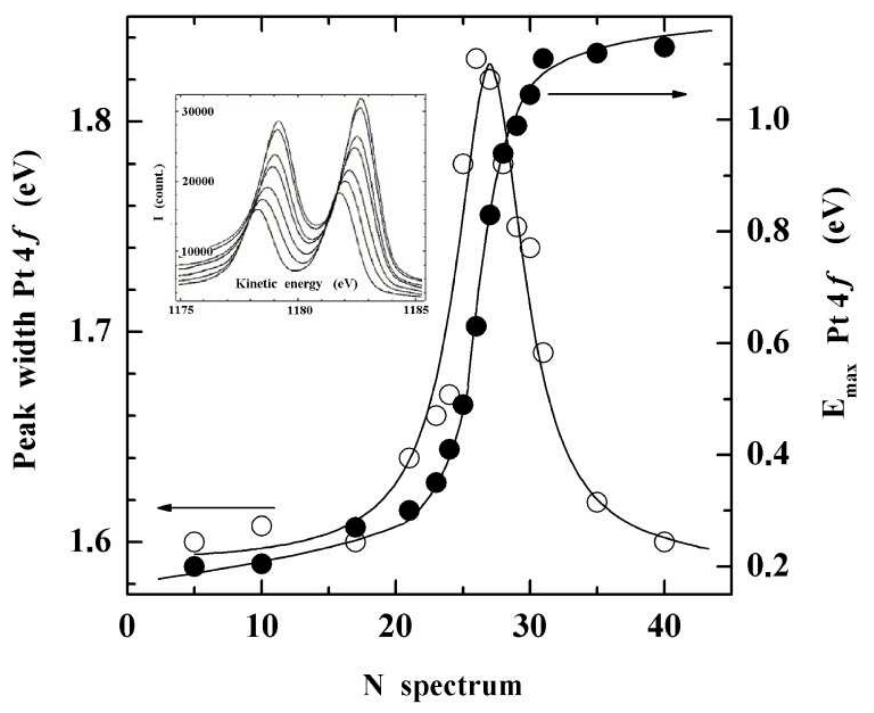

Fig. 9. Changes during the annealing of a $15.6 \mathrm{~nm}$ Pt layer at $400^{\circ} \mathrm{C}$ versus spectrum number: position of $\mathrm{Pt} 4 \mathrm{f}$ peak (right scale, open circles); peak width (left scale, solid circles).

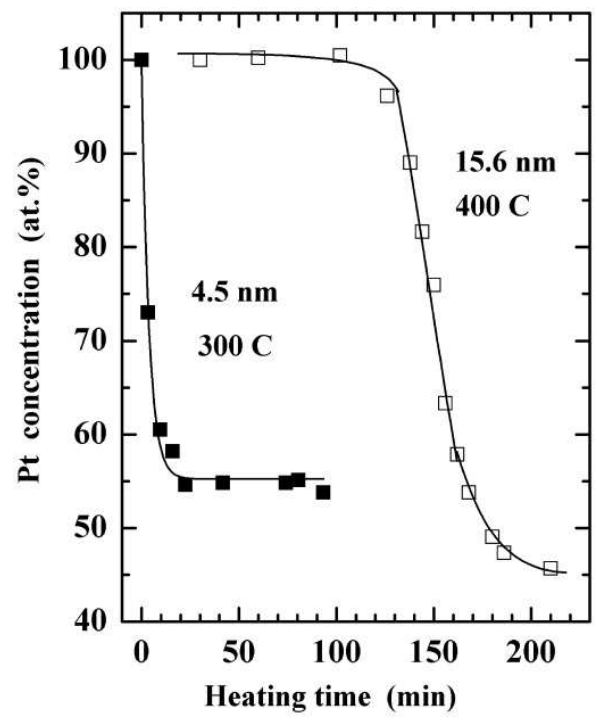

Fig. 10. Platinum surface concentration versus annealing time: $4.5 \mathrm{~nm}$ thick Pt layer annealed at $300^{\circ} \mathrm{C}$ (solid boxes); $15.6 \mathrm{~nm}$ layer annealed at $400^{\circ} \mathrm{C}$ (open boxes).

Subsequent X-ray structure analysis of specimens cross-section showed that resulting polycrystalline film had MnP type orthorhombic structure with the following constants: $a=$ $0.592 \mathrm{~nm}, b=0.558 \mathrm{~nm}, c=0.360 \mathrm{~nm}$, which corresponds to the lattice parameters of PtSi compound (Suprun et al., 1995). 
There is one more interesting moment in this technology, which is not going to be considered here, but should be considered in atomic layer engineering. The point is that the exposition of the obtained compound to the atmosphere changes the chemical composition of its surface due to formation of silicon oxides. Oxygen is a chemically active element, and breaking up of platinum silicide leads to a number of unwanted effects.

Thus, the use of the XPS method gives us a clear idea of chemical reactions on the surface of a solid body, which helps optimise manufacturing techniques of objects with desired properties.

\section{Formation of $\mathrm{GaAs} / \mathrm{Ge}$ heterointerface in the presence of intrinsic GaAs oxides}

Application of MBE method in manufacturing of heterostructures means use of chemical reactions in solid phase having very their own specific features. For instance, growth of the epitaxial layer takes place in nonequilibrium conditions. In epitaxy the ratio between the flow of the material from the source, determined by its temperature, and the pressure of the equilibrium vapour of that material over the substrate surface may be more than $10^{10}$ times higher! One example is the obtaining of Ge epitaxial layers (melting temperature $938^{\circ} \mathrm{C}$ ) on GaAs substrate at substrate temperature (Ts) $200^{\circ} \mathrm{C}$. Obviously such estimation of the system deviation from equilibrium conditions is not correct. The matter is that the crystal lattice of Ge is built from $s p^{3}$ hybridized atoms, and the life time of such excited state during the evaporation of material according to some estimations reaches $10^{-3} \mathrm{~s}$ (Davey et al., 1963). The duration of flight of the adatom or cluster from the evaporator to the substrate in MBE chamber is significantly smaller than this value. Thus, hybridized atoms ready to be embedded into the crystal lattice come to the substrate surface. Their initial energy is not determined by the temperature of substrate, but it will determine the exited state relaxation time. On the whole, stoichiometry of the reaction product in solid phase, which is characterized by crystal perfection of the grown layer, depends on the selected technological mode.

However, there is one more feature related to the formation of the heterointerface. Boundary conditions not only require adherence to stoichiometry, since the excessive component interferes with crystal lattice periodicity, but they also require coordination of atomic dipoles on the boundary (Gleim et al., 2003; Pashley, 1989). All that leads to such effects as "floating" - arrival of not embedded component from the heterointerface to the growing layer surface, spatial degradation of the boundary, which is necessary for coordination of dipole moment, etc. Let us consider reception of heterojunction (HJ) GaAs-Ge as an example.

Nowadays this heterojunction is one of the most exhaustively studied. There is extensive literature reporting results of experimental studies of production processes, properties, and theoretical models describing characteristics of an ideal heterojunction and deviations from them occurring in the presence of different imperfections and defects. Such heterostructure is notable for the same type of symmetry of crystal lattices on both sides of the interface and perfectly matching lattice periods. Distinctions caused by the type of valency bond in GaAs and Ge are studied in great detail both theoretically and experimentally. It was shown that during formation of the heterointerface a transitive mixed composition layer is formed on 
GaAs polarized faces, which matches atomic dipole potential. There is a good agreement of theoretical data on the value of the valence band discontinuity in those semiconductors with measured values, with a certain spread in observed data (Franciosi \& Walle, 1996; Yu et al., 1992). This may be caused by the fact that when a compound is used as a substrate, the composition on the free surface always differs from the bulk stoichiometry, which complicates the processes of interface formation.

The structure and morphology of the intrinsic GaAs oxide which plays an essential role in the technology of heterojunction production are also studied in great detail. According to the phase diagram, the thermodynamic equilibrium with GaAs under normal conditions is attained by the combination of limiting oxide $\mathrm{Ga}_{2} \mathrm{O}_{3}$ and arsenic (Torkhov, 2003). In this connection, processes of arsenic oxides reduction and $\mathrm{Ga}_{2} \mathrm{O}_{3}$ oxide cluster formation (Van Buuren at all., 1991) will be observed in the oxide formed during chemical processing with participation of the monocrystal surface. Substrate heating in vacuum causes desorption of arsenic and its oxides and formation of loose coating with possible lacunas. At the next stage of clearing in the presence of fragments of free GaAs surface one may observe a reaction leading to reduction of valency of Ga in the limiting oxide and desorption of components at temperatures about $500^{\circ} \mathrm{C}$ (Carli \& Bianchi, 1994; Isomura et al., 2007)

$$
\mathrm{Ga}_{2} \mathrm{O}_{3}(\mathrm{~s})+4 \cdot \mathrm{GaAs}(\mathrm{s}) \Rightarrow 3 \cdot \mathrm{Ga}_{2} \mathrm{O} \uparrow+2 \cdot \mathrm{As}_{2} \uparrow
$$

Thus, on GaAs surface during heating one may observe a complex chain of chemical reactions leading to the removal of intrinsic oxides of the material and loss of some stoichiometric arsenic. It was shown that the aforementioned system is very sensitive to the method of oxide preparation and to its initial thickness (Yamada \& Ide, 1995). Because of the low pressure of $\mathrm{Ga}_{2} \mathrm{O}_{3}$ vapours the time (or temperature) necessary for completion of the second stage of surface cleaning, accompanied by reduction of Ga valency, is determined by the initial oxide thickness. It should not be overlooked, that the reaction with participation of GaAs only goes intensively in the presence of free surface which could be not so easily accessible in case of thick oxide. One of the drawbacks of complete removal of GaAs oxides by means of thermal heating in vacuum is certainly coarsening of the surface and its enrichment with gallium. During subsequent deposition of material, it leads to the formation of various defects in the interface zone and "tails" of background impurities penetrating into the growing layer (Kawanaka \& Sone, 1990). Therefore the usual procedure is to grow the buffer GaAs layer on the substrate in order to restore the structure and relief of the surface. However, even in this case there still can be a wide spread in electronic properties and electrophysical parameters of the heterojunction, caused by the presence of a superstructure with its own Ga to As ratio on the substrate surface during epitaxy.

Earlier we used a method of scattered laser radiation to study desorption of intrinsic GaAs oxide during its heating in vacuum (Suprun et al., 1997). It was shown that it is possible to obtain island-like Ge films on GaAs substrate in the intrinsic oxide lacunas in case of incomplete surface cleaning. Moreover, using reflection high energy electron diffraction (RHEED), we observed a formation of GaAs-Ge heterointerface under the conditions of incomplete oxide removal from the substrate surface, which is described below.

Standard semi-insulating and $\mathrm{n}+\mathrm{GaAs}(001)$ wafers $50 \mathrm{~mm}$ in diameter were used as specimens after their chemical processing (Suprun \& Fedosenko, 2009). The wafers were 
fixed on a molybdenum carrier, loaded into the load lock of MBE installation and then vacuumed. After its transfer to the growth chamber the specimen was heated up to $500^{\circ} \mathrm{C}$ and Ge was deposited at the rate less than $0.5 \mathrm{~nm} \times \mathrm{min}^{-1}$. RHEED method allowed us to observe formation of the atomically-smooth epitaxial layer with $2 \times 2$ superstructure simultaneously with the removal of remaining gallium oxides (Fig. 11). Note that formation of $\mathrm{GeO}$ and its desorption do not take place in this case because of the low substrate temperature.

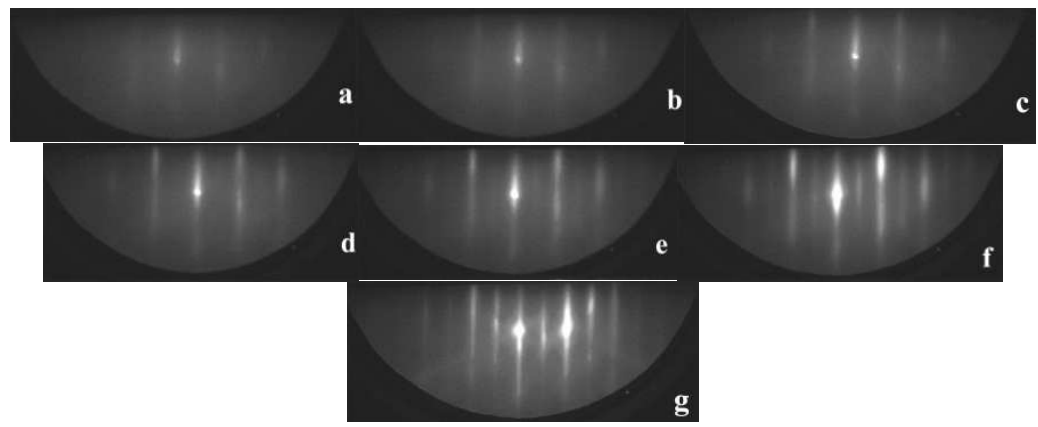

Fig. 11. Successive changes in the diffraction pattern observed during deposition of Ge onto $\operatorname{GaAs}(001)$ at $500^{\circ} \mathrm{C}$. (a) The initial surface in direction [110] before deposition; after the deposition of the Ge layer with a thickness of $(\boldsymbol{b}) 0.4 \mathrm{~nm},(\boldsymbol{c}) 1.2 \mathrm{~nm},(d) 1.5 \mathrm{~nm},(\boldsymbol{e}) 2.0 \mathrm{~nm},(f)$ $2.5 \mathrm{~nm}$, and $(g) 10.5 \mathrm{~nm}$.

As it follows from Fig. 11, one of the features of the growth process under these conditions is that $2 \times 2$ superstructure is formed gradually and finishes when the layer is several nanometers thick. It can be caused by the fact that the growth begins in the oxide lacunas, and it seems as if the picture is "developing" through the diffuse background. By contrast the reconstruction on a completely cleaned GaAs surface is only observed after deposition of 2 germanium monolayers.

Two factors are critical for this mode of epitaxy: the first is the chemical preparation of the specimen during which a thin intrinsic GaAs oxide is formed, and the second is the rate of Ge deposition during formation of heterointerface. Non-compliance with these conditions leads to formation of polycrystalline Ge layer (Suprun \& Fedosenko, 2009).

The following experiment was developed to study the mechanisms of the observed process. After chemical treatment, $n+G a A s$ plate (001) was loaded into the analysis chamber. XPS spectra were acquired of GaAs surface before and after heating of the specimen to $500^{\circ} \mathrm{C}$. Al $\mathrm{K}_{\alpha}$ line with energy of $1486.6 \mathrm{eV}$ was used as the source, spectra were acquired with $0.6 \mathrm{eV}$ resolution and angle of acquisition 45 and $70^{\circ}$ off the normal to the plate surface. Analysis depth ratio for these acquisition angles was 2:1, respectively. Figures 12 and 13 show As and Ga spectra, and the distance between peaks, corresponding to bulk components, was 21.9 $\mathrm{eV}$. The following table shows parameters of Ga 3d and As 3d doublets, namely, values of the spin-orbital splitting, components ratio in the doublet, values of full width at half-height of Gauss and Lorentz functions used to fit the experimental spectra, energy shifts of various compounds of $\mathrm{Ga}$ and $\mathrm{As}$ and their surface components in relation to their bulk components. 


\begin{tabular}{|l|l|l|}
\hline Peak & $\begin{array}{l}\text { Ga } \\
3 d\end{array}$ & As 3d \\
\hline Spin-orbital splitting (eV) & 0.45 & 0.69 \\
\hline Components ratio & 1.5 & 1.4 \\
\hline Gaussian width $(\mathrm{eV})$ & 0.6 & 0.6 \\
\hline Lorentzian width $(\mathrm{eV})$ & 0.7 & 0.7 \\
\hline Surface component S1 (eV) & 0.34 & -0.43 \\
\hline Surface component S2 (eV) & -0.32 & \\
\hline Chemical shift - As (eV) & & 0.6 \\
\hline $\mathrm{As}_{2} \mathrm{O}_{3}$ & & 3.2 \\
\hline $\mathrm{Ga}_{2} \mathrm{O}$ & 0.4 & \\
\hline $\mathrm{Ga}_{2} \mathrm{O}_{3}$ & 1.5 & \\
\hline
\end{tabular}

Table 1.

Values of spin-orbital splitting, components ratio in the doublet and energy shifts for surface components shown in the table were taken from (Lay et al., 1991), and the chemical shifts values ware taken according to (Holloway \& McGuire, 1995). Initial state of the surface matches contemporary views on formation of intrinsic GaAs oxide. For example, As 3d peak (Fig. 12 above) can be shown as a combination of peaks of pure arsenic, the component corresponding to As-Ga $(\boldsymbol{B})$ bulk bond, and relatively small peak of $\mathrm{As}_{2} \mathrm{O}_{3}$ oxide. At the same time initial condition of $\mathrm{Ga}$ (Fig. 13 above) is characterized by the presence of $\mathrm{Ga}_{2} \mathrm{O}$, $\mathrm{Ga}_{2} \mathrm{O}_{3}$ oxides, Ga-As $(\boldsymbol{B})$ bulk components and not so pronounced surface components $S_{1}$ and $S_{2}$ (this component is not marked in the top part of Fig. 13).
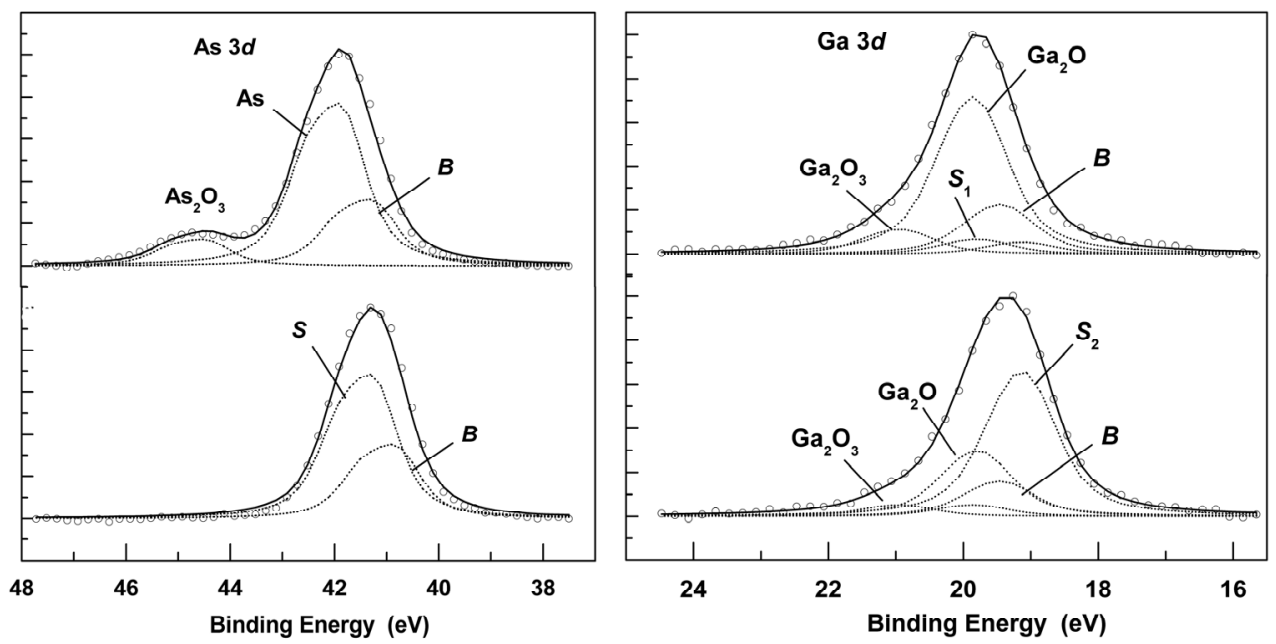

Fig. 12. (right). Spectra of Ga 3d, obtained immediately after chemical processing of the specimen (above), and after its heating to $500^{\circ} \mathrm{C}$ (below). Symbols on the spectra correspond to the table and Fig. 12. 
Fig. 12 (left). Spectra of As $3 \mathrm{~d}$ obtained immediately after chemical processing of the specimen (above), and after its heating to $500^{\circ} \mathrm{C}$ (below). Experimental data on the spectra are marked with circles, dashed lines correspond to fitting components, and the continuous line shows the sum of all fitting components. Symbols on the spectra: $\boldsymbol{B}$ - is a component corresponding to As-Ga bulk bond, $S$ is a surface component, As is elemental arsenic.

When the specimen is heated to $500^{\circ} \mathrm{C}$, one may observe the complete desorption of arsenic and its oxide (Fig. 12 below). The registered peak is the sum of As-Ga volume and surface components. Significant changes are observed in Ga 3d peak (Fig. 13 below). Content of $\mathrm{Ga}_{2} \mathrm{O}, \mathrm{Ga}_{2} \mathrm{O}_{3}$ oxides decreases and surface component $S_{2}$ corresponding to low-coordinated $\mathrm{Ga}$ becomes dominating in the spectrum. Presence of gallium with dangling valency bonds, hence being in chemically active state, should facilitate the reaction development according to formula (1). If at this time we begin deposition of germanium onto the surface, it, presumably, will lead to binding of released arsenic, which prevents disturbance of surface stoichiometry and its enhancement with gallium.

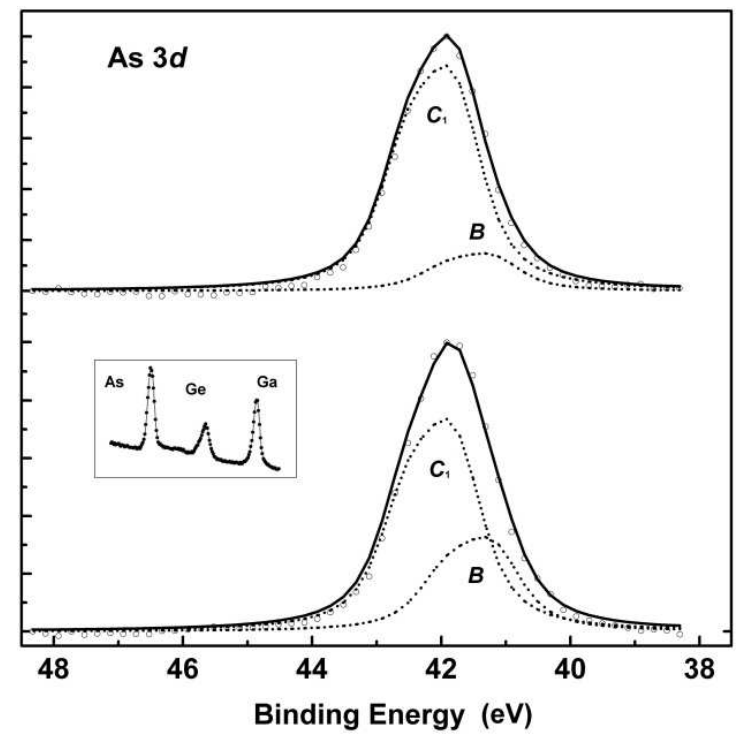

Fig. 13. As $3 \mathrm{~d}$ spectra obtained after Ge deposition at photoelectrons acquisition angle of $70^{\circ}$ (above) and $45^{\circ}$ (below) off the normal to the plate surface, the insert shows summarizing spectrum. Components symbols are the same as in figures above.

Analytical installation was equipped with a growth chamber which allowed us to obtain the required structure and to transfer the specimen into the analysis chamber without its contact with the atmosphere. The research in situ technique was similar to the one used to study low temperature recrystallization of Ge on ZnSe and is described in (Suprun \& Fedosenko, 2007). Ge layer was deposited at room temperature on a substrate with surface structure shown in Fig. 12 and 13 after it was heated up to $500^{\circ} \mathrm{C}$. Then the specimen was transferred to the analysis chamber where it was consecutively heated to $500^{\circ} \mathrm{C}$, with a step of $100^{\circ} \mathrm{C}$, and its surface spectra recorded. 
Attention must be paid to significant difference between the situation with the formation of Ge-GaAs heterostructure in MBE installation and the modelling experiment carried out in the analytical installation. Deposition of Ge in MBE installation began at low rate at $500^{\circ} \mathrm{C}$ after removal of arsenic oxides, and in analytical installation Ge layer about $1 \mathrm{~nm}$ thick was deposited on similarly prepared substrate surface at room temperature. Hence, in the second case Ge layer blocked the substrate surface, complicating interaction of the components in the heterosystem and their subsequent desorption. For us it was important to observe basic changes of chemical state of elements in the interface zone and the surface.

Fig. 14 shows As 3d spectra, obtained immediately after deposition of Ge onto the substrate at room temperature. Note, that in contrast to Fig. 12 there is no surface arsenic component, and the integral spectrum only consists of two components. One of them - the bulk - is more pronounced at greater depth of the analysis (angle - $45^{\circ}$ off the normal to the surface), another one, $\left(C_{1}\right)$ - behaves quite the opposite. Component $C_{1}$ is shifted by $0.6 \mathrm{eV}$ in relation to the bulk component, $\mathrm{B}$, and the observed dependence on the analysis depth indicates that it is located closer to the surface. This component (As on Ge) may be related to a well studied process of Ge surface energy reduction because a part of arsenic comes to the surface from the interface (Monch \& Grant, 1980; Bauer, 1983). The reaction is activated due to high energy of Ge adatoms coming from the source during the deposition.

There is one more interesting point: the change of the structure (not the composition) of the surface layer may be traced by As and Ga spectra dependences on temperature: heating of the specimen leads to a sharp increase of volume component at $\mathrm{Ts} \approx 300^{\circ} \mathrm{C}$ if photoelectrons are registered at $45^{\circ}$ off the normal to the surface (Fig. 15). This figure shows temperature behaviour of bulk components $(B)$ contribution (in relative units) to the total peak area of the respective element at two acquisition angles. Such difference in the behaviour of this component may be due to crystallisation of initially amorphous Ge layer deposited at room temperature. This process enables possible local reduction of Ge thickness at grain boundaries, which leads to increased flux of photoelectrons from the substrate. At a flat acquisition angle $-70^{\circ}$ off the normal - this effect is less pronounced due to shadowing of these areas by relief roughness. Subsequent reduction of bulk components contribution to the spectrum reflects smoothing of the surfaces with temperature due to diffusion.

Rise in the specimen temperature occurs simultaneously with the process of reduction of gallium valency in oxide and its desorption from the surface. As can be seen from Fig. 9, the basic component in gallium spectrum is $\mathrm{Ga}_{2} \mathrm{O}$ oxide. Registered reduction of this component at $300^{\circ} \mathrm{C}$ occurs as a result of increased contribution of the bulk component described above. Near $500^{\circ} \mathrm{C}$, the process of $\mathrm{Ga}_{2} \mathrm{O}$ oxide desorption goes intensively despite the fact that the presence of $\mathrm{Ge}$ at the layer surface could complicate the situation.

Fig. 15 (left). Relative area of bulk component (B) in spectra of As (circles) and Ga 3d (triangles), measured at $70^{\circ}$ (white) and $45^{\circ}$ (black) off the normal to the surface as a function of temperature.

Sudden change in the composition of As $3 \mathrm{~d}$ peak is observed at the same temperature of $500^{\circ} \mathrm{C}$ : there appears another arsenic compound with germanium. The peak fitting component designated as $C_{2}$, is shifted by $0.8 \mathrm{eV}$ in relation to the bulk component $\boldsymbol{B}$ (Fig. 17). The similar state of arsenic is described in (Stucki et al., 1983) for germanium arsenide 
(GeAs) monocrystal. It is safe to assume, that the saturation degree of arsenic bonds at GaAs-Ge heterointerface is higher than that on Ge surface, and the value of chemical shift is respectively greater. Absence of the bulk component in the spectrum indicates high thickness uniformity of germanium layer at the final stage of heating.
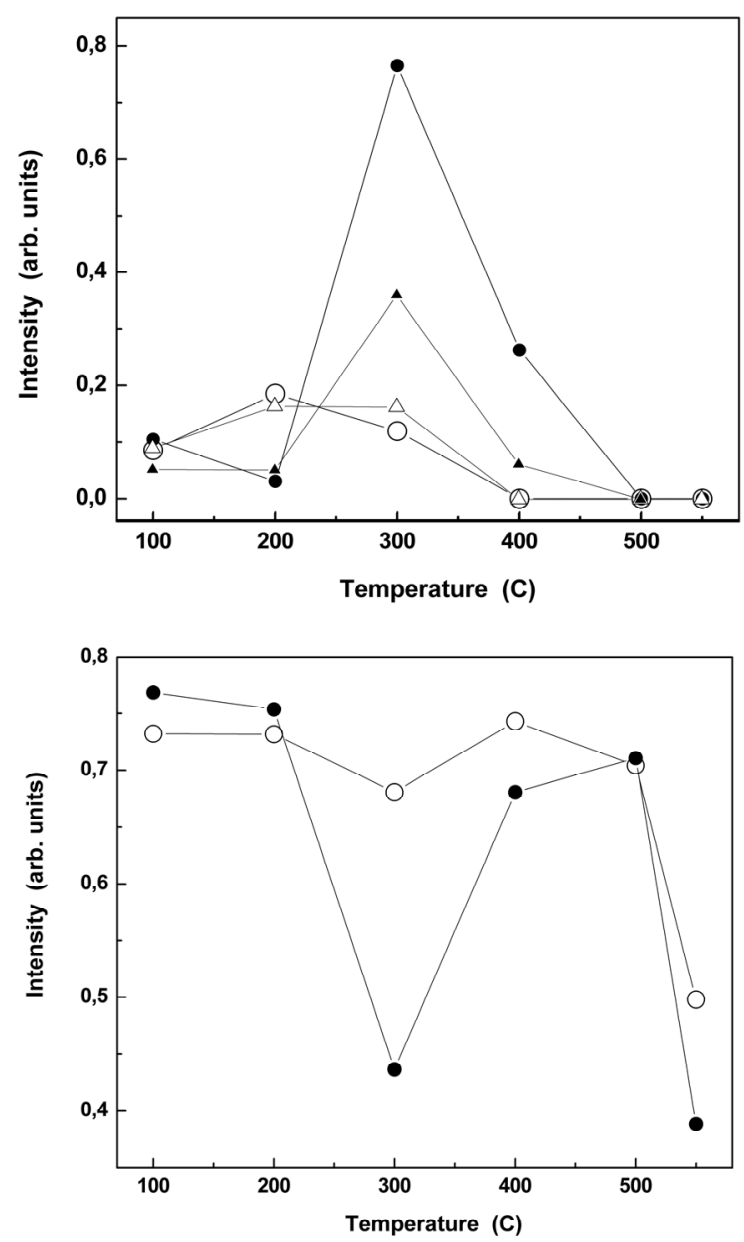

Fig. 14. (right). Relative area of $\mathrm{Ga}_{2} \mathrm{O}$ component, measured at $70^{\circ}$ (white circles) and $45^{\circ}$ (black circles) off the normal to the surface as a function of temperature.

It is informative to compare dependences between charge carriers concentration and thickness of Ge layers grown on the surface of semi-insulating GaAs, stabilized by Ga, and without removal of oxide layer at $\mathrm{T}_{\mathrm{S}}=500^{\circ} \mathrm{C}$. In the second case, after the formation of the interface zone, the growth rate increased up to $0.3 \mathrm{~nm} \times \mathrm{sec}^{-1}$. Layers thickness was $\approx 2$ micron, then Hall structures were formed on the specimens using photolithography, which were measured during the gradual etching of Ge. Calculations of charge carriers concentration and mobility were made for the etched layer; the results are shown in Fig. 18. 


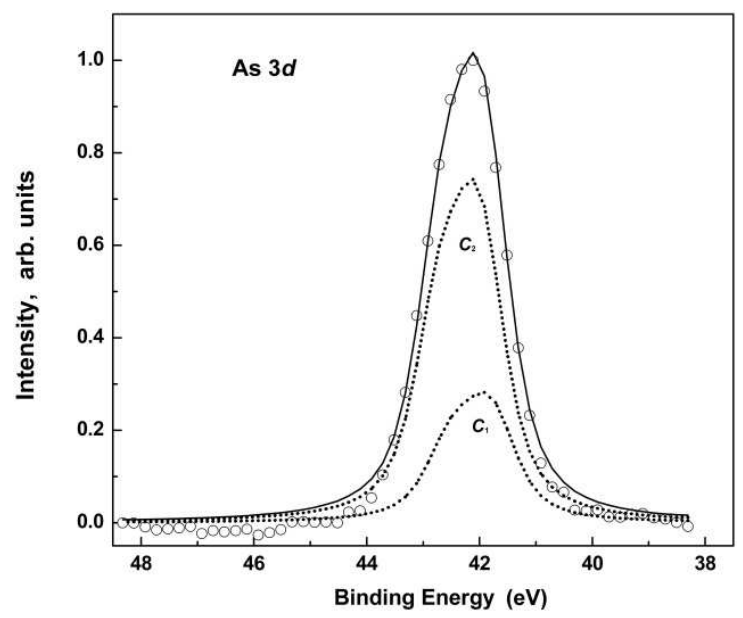

Fig. 15. As 3d spectrum of the surface obtained after deposition of $\mathrm{Ge}$ and heating of the specimen up to $500^{\circ} \mathrm{C}$, dashed lines show components $C_{1}$ and $C_{2}$ (As-Ge compounds on the surface and at the heterointerface).

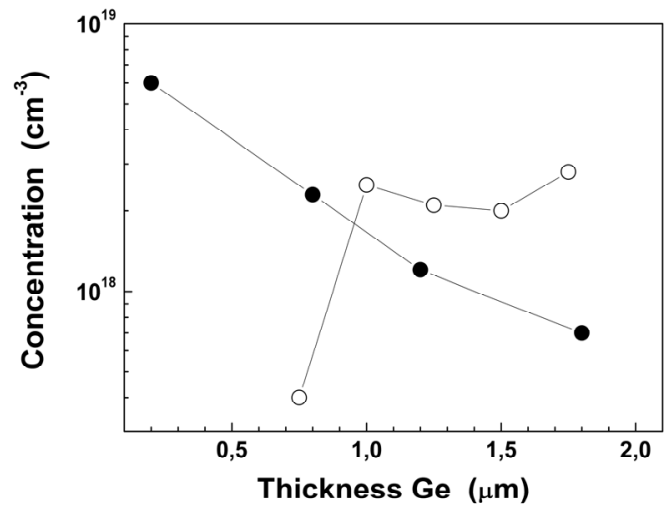

Fig. 16. Dependences between hole concentration and films thickness calculated for etched away layer: black circles - Ge grown on Ga stabilized substrate surfaces, open circles show low temperature epitaxy.

In both cases p-type conductivity layers were obtained, but dependences between concentration and thickness had different behaviour. During the deposition onto substrate surface with the superstructure stabilized by gallium, there is a gradual embedding of the excessive component into the growing layer (Ga solubility limit in Ge is about $10^{20} \mathrm{~cm}^{-3}$ ). As a result one can observe the impurity concentration profile growing towards the heterointerface. In the second case concentration in the boundary zone is rather small and increases with the film thickness with a rise in the growth rate during epitaxy. This indicates existence of another source of background impurity, which means that the interface cannot obviously be such source. Measured Hall mobility of charge carriers on etched away layer was in the range of $200-350 \mathrm{~cm}^{2} \times \mathrm{V}^{-1} \times \mathrm{s}^{-1}$ and corresponded to its value in bulk Ge 
monocrystal with the same impurity concentration. It indicates high structural perfection of the obtained epitaxial films.

Thus, application of XPS method allows us to understand complex phenomena of formation of heterointerface, and to optimize the technological processes. Reliable information often can only be obtained using various research methods supplementing each other. This is why here and further on we will present some results obtained using other techniques, which helps studying the peculiarities of the course of reactions in solid phase.

\section{Preparation and analysis of $\mathrm{ZnSe} / \mathrm{Ge}$ structures on GaAs substrate}

Problems similar to those described above also occur during formation of GaAs-ZnSe heterojunction. In our work (Suprun et al., 2009) we described peculiarities of this heterointerface formation, related to ZnSe nutrient being used as a source during the growth. Crystal lattices of both materials in this heterojunction are also of the same type and have very similar constants. The spatial symmetry on both sides of the boundary between GaAs-ZnSe is not discontinued; the coordination number remains the same, it is only the dipole value between the nearest neighbours that changes. This respectively causes formation of a transitive layer from the elements composition (III-VI and II-V), matching the charge distribution at the boundary, as it was described, for example, in (Schmeißer et al., 1999; Pan et al., 2002). In this case the complete lattice period has on average 4 electrons per lattice point. But if there is any deviation from bulk stoichiometry on the substrate surface owing, for example, to the presence of a superstructure, then again there will be a problem with the excessive element embedding.

We used epitaxial layers of ZnSe on GaAs as a substrate in formation of an array of mechanically unstressed quantum dots (QD) of Ge (Neizvestny et al., 2001; Talochkin et al., 2005). XPS method was used for direct observation of recrystallization of germanium amorphous layer. This process is accompanied by the change of coordination number in the examined structure, which characterizes the average number of the dangling valence bonds per atom. In other words, the smaller is the size of the clusters, the greater is the ratio of the surface layer to volume, and the larger is the number of atoms with non-saturated bonds. Recrystallization is usually attributed to the phase changes, but from the XPS method point of view, the observed changes in binding energy of the analyzed element are absolutely similar to those occurring in chemical reactions. We may look at the term stoichiometry in a slightly different way if we mean the saturation degree of valency bonds. For a monocrystal of some compound, for example ZnSe or GaAs, compositional disorders are directly connected with the broken spatial symmetry. For a covalent semiconductor, such as Ge or $\mathrm{Si}$, everything will be the same, though it is not so obvious, since the element composition does not change.

Analysis of physical properties of a material in the form of nanoclusters is now a very topical and often rather complicated problem. With the objects of such a size, the measurement method must be extremely "delicate" in order not to introduce systematic error caused by interaction with the device. In this connection of special interest are works by Chang Sun et al. (Sun, 2007), offering a model of bond-order-length-strength (BOLS) correlation mechanism. BOLS model makes it possible to examine dependences between the size and form of a nanoobject and such values as shift of core levels, width of the band gap, 
temperature of phase transitions, etc. It is based on the principle that the only factor determining all properties of a solid body is the energy of interatomic interaction; the basic atomic characteristic in the crystal lattice is the coordination number $(\mathrm{CN})$, characterizing the number of nearest neighbours; existence of dangling valency bonds causes $\mathrm{CN}$ reduction; the spatial size of the dangling electronic orbital is reduced, which leads to the increase in energy per $\mathrm{CN}$ (though the total binding energy of atom falls due to $\mathrm{CN}$ reduction). Finally, reduction of the object size leads to higher ratio between volume of the surface shell, being no more than 3 monolayers thick, to the total volume, which respectively increases the role of the surface in the examined physical properties. It is clear, that existence of dangling bonds leads to a change in atom energy in the lattice, including change of the position of internal core levels and all parameters of the lattice caused by the change of binding energy with neighbours. Comparison between calculations and experimental data in those works shows good agreement, and it is offered for a wide range of materials, for which small size clusters were obtained. However, there are still no data for such semiconductor as germanium.

We previously described some methods used to obtain an array of unstrained Ge quantum dots (QD) on heteroepitaxial ZnSe layers. The examined ZnSe/Ge heterosystem is characterized by similar type of the lattice and small mismatch value for their constants, $\approx$ $0.2 \%$, which excludes occurrence of the mechanical stress in the substrate-nanoobject system. When studying the islets formation in this system, we observed a low-temperature recrystallization of $\mathrm{Ge}$ - the transition from the amorphous state to the polycrystalline one, and then into the continuous epitaxial layer (Suprun \& Fedosenko, 2007). The temperature was significantly below the melting temperature of the bulk material. Later we conducted experiments in situ for direct observation of this process using RHEED and XPS methods, and the results were analyzed in terms of BOLS model.

XPS method was used to acquire the spectra shown in Fig. 19 (the insert shows RHEED patterns, obtained when a similar specimen was heated in $\mathrm{MBE}$ chamber). $\mathrm{Al} \mathrm{K}_{\alpha}$ line was used as the radiation source. From the growth chamber through the air lock the carrier was transferred into the analysis chamber, where spectra were recorded. After deposition of a $1 \mathrm{~nm}$ thick germanium layer, the specimen was heated step-by-step, and XPS spectra were recorded for each step. As can be seen from the Fig. 19, the distance between $\mathrm{Zn} 3 \mathrm{~d}$ and Se $3 \mathrm{~d}$ peaks after Ge deposition, and during subsequent heating of the specimen from room temperature to $623 \mathrm{~K}$, changes only slightly, no more than by $0.10 \mathrm{eV}$, which remains within the error limit of peak position measurements. This finding proves that there is no significant change in chemical bonding between $\mathrm{Zn}$ and Se in the presence of Ge. At the same time the initial position of Ge 3d peak after deposition (the curve on Fig. 19, corresponding to the temperature of $293 \mathrm{~K}$ ) is shifted in relation to the position in the bulk material towards higher binding energies. Increase in binding energy of core peaks is typical for nanoscale clusters (Howardet al., 2002; Nanda al., 1999). During further heating of the specimen one may observe significant shift of Ge 3d level by more than $1 \mathrm{eV}$ towards lower binding energies.

To find the dependence of Ge 3d level shift on temperature we chose the position of Se $3 d$ peak as a reference point. Both peaks underwent the background subtraction and were fitted using Gauss function, which allowed us to measure Ge $3 \mathrm{~d}$ shift with error of \pm 50 meV. Fig. 20 shows the dependence of energy difference between Se $3 \mathrm{~d}$ and Ge $3 \mathrm{~d}$ peaks on specimen temperature, and the dependence of the value of Ge $3 \mathrm{~d}$ peak dispersion on 
temperature. It can be seen, that with a rise in specimen temperature there is a shift of the core level towards lower binding energies, and the curve gets closer to saturation near 450 $\mathrm{K}$. According to the reference data, the difference in binding energy between Se $3 \mathrm{~d}$ and $\mathrm{Ge}$ $3 \mathrm{~d}$ levels in bulk ZnSe and Ge materials is $25.25 \mathrm{eV}$. Fig. 20 shows, that peak position even in the final state does not reach the limiting value corresponding to bulk materials, which is due to small thickness of the forming layer.

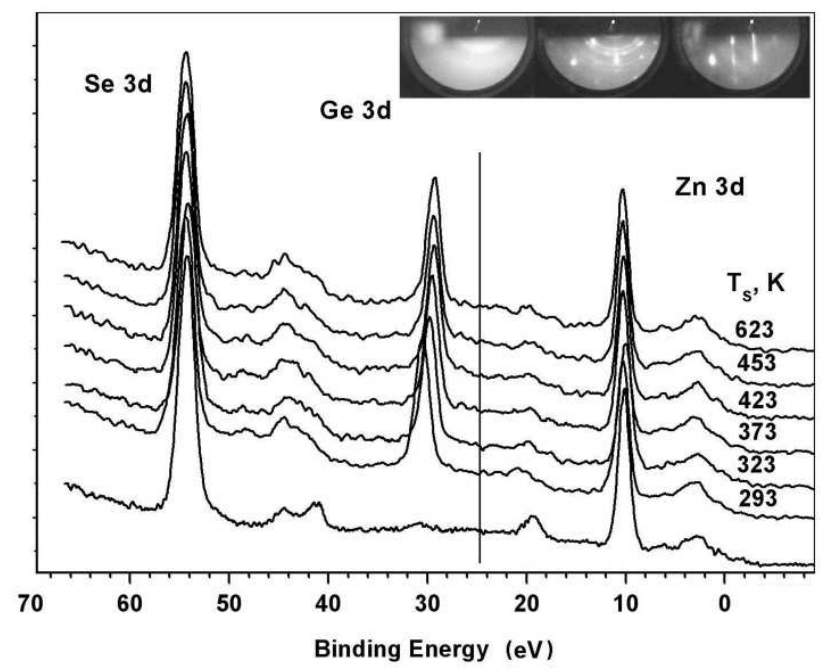

Fig. 17. Changes of XPS spectra of GaAs/ZnSe/Ge surface with temperature, the lowermost - before Ge deposition. On the inset, changes in RHEED patterns are shown, occurring during the heating of the crystal structure of Ge layer $\approx 1 \mathrm{~nm}$ thick deposited at room temperature on ZnSe epitaxial film.

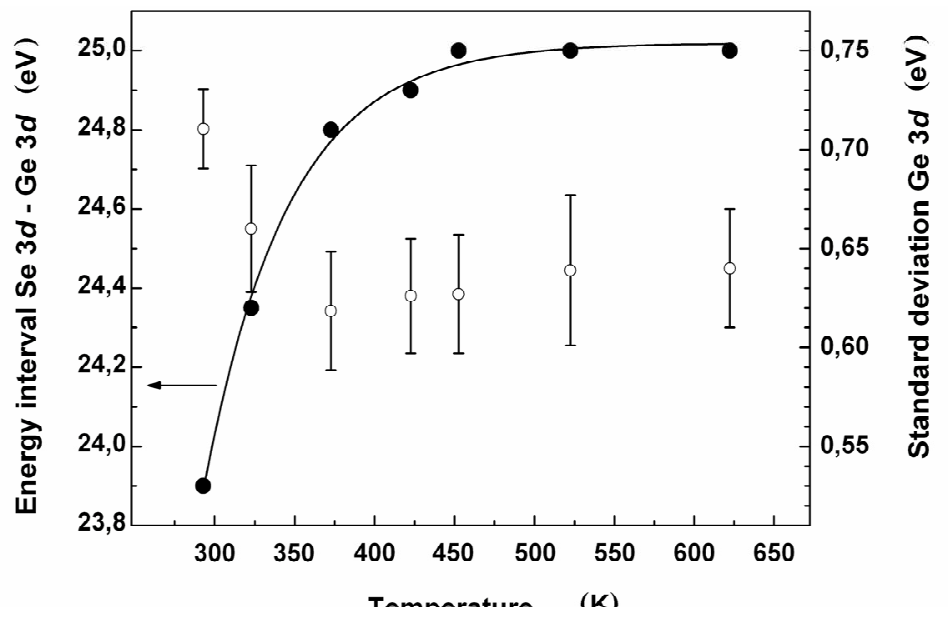

Fig. 18. Energy difference between Se $3 d$ and Ge $3 d$ peaks as a function of the specimen temperature (left), dependence of the value of $\mathrm{Ge} 3 \mathrm{~d}$ peak dispersion on temperature (right). 
Let's now describe the observed changes of Ge $3 \mathrm{~d}$ level position in terms of BOLS model, assuming that there are clusters about $1 \mathrm{~nm}$ in the amorphous layer and the interaction between the material and substrate is weak.

Value of core level energy shift (Sun et al., 2001; Sun, 2004) can be described by the formula:

$$
\mathrm{E}_{3 \mathrm{~d}}(\Delta)-\mathrm{E}_{3 \mathrm{~d}}(1)=\left[\mathrm{E}_{3 \mathrm{~d}}(\infty)-\mathrm{E}_{3 \mathrm{~d}}(1)\right](1+\Delta)
$$

where $E_{3 \mathrm{~d}}(\Delta)$ is the core level energy for nanoobject; $E_{3 \mathrm{~d}}(1)$ is the energy corresponding to that level position in one isolated atom, $E_{3 \mathrm{~d}}(\infty)$ is the level energy in bulk material. Value $\Delta$ describes surface contribution, determined by nanoobject type and dimensions. $\Delta$ is a product of $\gamma_{i}$ factor $(i \leq 3$, layer number counted from the surface), corresponding to the ratio between the surface and the volume for different nanoobjects, depending on their dimensions multiplied by relative change of bond length $\left(c_{i}=d_{i} / d_{0}\right)$, caused by reduction of $\mathrm{CN} z_{i}$.

$$
\begin{aligned}
& \Delta=\sum_{\mathrm{i}} \gamma_{\mathrm{i}}\left(\mathrm{c}_{\mathrm{i}}^{-\mathrm{m}}-1\right), \\
& \mathrm{c}_{\mathrm{i}}=\mathrm{d}_{\mathrm{i}} / \mathrm{d}_{0}=2 /\left[1+\exp \left[\left(12-\mathrm{z}_{\mathrm{i}}\right) / 8 \mathrm{z}_{\mathrm{i}}\right]\right] \\
& \gamma_{\mathrm{i}}=\frac{\mathrm{N}_{\mathrm{i}}}{\mathrm{N}}=\frac{\mathrm{V}_{\mathrm{i}}}{\mathrm{V}}=\left[4 \pi((\mathrm{L}-(\mathrm{i}-1)) \mathrm{d})^{2} \mathrm{~d}_{\mathrm{i}}\right] /\left(\frac{4 \pi}{3}(\mathrm{Ld})^{3}\right)=\frac{3}{\mathrm{~L}}\left(1-\frac{\mathrm{i}-1}{\mathrm{~L}}\right)^{2} \frac{\mathrm{d}_{\mathrm{i}}}{\mathrm{d}}
\end{aligned}
$$

In these equations, $L$ is the number of atoms fitting along radius $L=D / 2 d_{0}$ ( $D$ is cluster diameter or cross-section of quantum wire, or thickness of continuous layer). Generally speaking, for small size objects it is necessary to take an average $d$ value which will differ from $\mathrm{d} 0$. Total atom $\mathrm{CN} z_{0}$ for Ge lattice (just like for $\mathrm{Si}$ and $\mathrm{C}$ - diamond) is 12, its value for the top cluster layer depending on radius, and it is determined by the formula: $z_{1}=4 \times(1-$ $0.75 / L)$.

Notably, fall in $\mathrm{CN} z$ from 12 to 8,6 and 4 leads to relative reduction of bond length by $3 \%$, $4 \%$ and $12 \%$, respectively. For ZnSe/Ge system, the difference in lattice constants about $\approx$ $0.2 \%$ in heterointerface zone should not considerably influence the characteristics of the layer material. Reduction of coordination number leads to reduction of relative length of ci bond in i-th layer according to (2) and to energy increase on $\mathrm{CN} z_{i}$. Such change can be calculated, using the adjustable parameter $\mathrm{m}$, which depends on material and is usually 1 for metals and about 4 for semiconductors. It was shown in (Sun, 2004), that good coordination between experimental data and calculations for clusters is obtained when $z_{1}$ for the top layer is defined according to equation (3), and for continuous layer with a flat surface $z_{1}=4$, and $\mathrm{CN}$ for the second and the third layers in both cases is taken as $z_{2}=6$ and $z_{3}=12$.

Relation between shifts of core Ge $3 \mathrm{~d}$ level at the beginning and at the end of recrystallization process will be:

$$
\frac{\mathrm{E}_{3 \mathrm{~d}}\left(\Delta_{1}\right)-\mathrm{E}_{3 \mathrm{~d}}(1)}{\mathrm{E}_{3 \mathrm{~d}}\left(\Delta_{2}\right)-\mathrm{E}_{3 \mathrm{~d}}(1)}=\frac{1+\Delta_{1}}{1+\Delta_{2}}
$$


In our case, as the initial state at temperature of $293 \mathrm{~K}$ we considered amorphous layer containing clusters 6 atoms in diameter, which corresponds to the average thickness of the initial layer. The final condition at $623 \mathrm{~K}$ is a continuous film 3 monolayers thick, taking into account consolidation of the structure during recrystallization and assuming that some large clusters do not melt. Values $\gamma_{i}$ for both cluster and layer and $d_{i} / d_{0}$ relation was calculated in a way similar to (Pan et al., 2002; Sun, 2004), for silicon in substitution of Ge bond length is $d_{0}=0.1366 \mathrm{~nm}$. Proceeding from the selected initial and final states using adjustment method, we found parameter $m=3.2$, provided that $\Delta E_{3 \mathrm{~d}}(\infty)=\left[E_{3 \mathrm{~d}}(\infty)-E_{3 \mathrm{~d}}(1)\right]=1.76 \mathrm{eV}$, i.e. it corresponds to the energy of $3 \mathrm{~d}$ level in single $\mathrm{Ge}$ atom $E_{3 \mathrm{~d}}(1)=27.2 \pm 0.1 \mathrm{eV}$, with reference value of its position in bulk material $E_{3 \mathrm{~d}}(\infty)=28.95 \mathrm{eV}$.

If we compare the obtained values for Ge with the data for silicon from (Sun, 2004), then for Si $m=4.88$, and energy difference for $\mathrm{Si} 2 \mathrm{p}$ core level is $\Delta E_{2 p}(\infty)=-2.46 \mathrm{eV}$. It is obvious, that the value of adjustable parameter for germanium is approximately one and a half times smaller than that for silicon.

Since the recrystallization process of an amorphous layer goes through a polycrystalline state, the cluster size must grow with temperature. This feature was used to check the value of adjustable parameter $m$. The table below shows $1+\Delta_{L}$ values calculated for each temperature of the specimen for matching radii $L$ and respective experimental relative change of Ge 3d peak position. Reference value of Se 3d peak position in $\mathrm{ZnSe}$ is $54.2 \mathrm{eV}$, and values of $E_{3 \mathrm{~d}}(1)$ and $\Delta E_{3 \mathrm{~d}}(\infty)$ for Ge were found above from the initial and the final state of the layer. Table 2 shows that we may observe a gradual increase in the average cluster size in the course of recrystallization (first column).

\begin{tabular}{|c|c|c|c|c|}
\hline $\begin{array}{l}\mathrm{L} \\
\mathrm{D} / 2 \mathrm{~d}\end{array}$ & $\begin{array}{l}\mathrm{T} \\
{[\mathrm{K}]}\end{array}$ & $\begin{array}{l}\Delta \mathrm{E}_{3 \mathrm{~d}}(\mathrm{~T})= \\
=\mathrm{E}(\mathrm{Se} 3 \mathrm{~d})-\mathrm{E}(\mathrm{Ge} 3 \mathrm{~d}) \\
{[\mathrm{eV}]}\end{array}$ & $\frac{E(\operatorname{Se} 3 d)-\Delta E 3 d(T)-E 3 d(1)}{-\Delta E_{3 d}(\infty)}$ & $1+\Delta_{L}$ \\
\hline 3 & 293 & 23.90 & 1.88 & 1.881 \\
\hline 4 & 323 & 24.31 & 1.64 & 1.582 \\
\hline 6 & 373 & 24.82 & 1.35 & 1.349 \\
\hline 7.7 & 423 & 24.98 & 1.26 & 1.261 \\
\hline 8 & 453 & 25.0 & 1.25 & 1.250 \\
\hline
\end{tabular}

Table 2.

It is safe to assume, that cluster growth passes through coalescence of small nucleating seeds, melting with a rise in substrate temperature. In the framework of the same BOLS model, it is possible to determine the cluster melting temperature, knowing its size and $\mathrm{m}$ parameter by the following equation:

$$
\frac{\mathrm{T}_{\mathrm{m}}(\mathrm{L})-\mathrm{T}_{\mathrm{m} 0}}{\mathrm{~T}_{\mathrm{m} 0}}=\sum_{3} \gamma_{\mathrm{i}}\left(\frac{\mathrm{z}_{\mathrm{i}}}{\mathrm{z}_{0}} \mathrm{c}_{\mathrm{i}}^{-\mathrm{m}}-1\right)
$$

where $T_{m}(L)$ is the melting temperature for a cluster of radius $L, T_{m 0}$ is the melting temperature of bulk material, and other designations are the same. Now, proceeding from the cluster size calculated from $3 \mathrm{~d}$ level shift, determined using XPS method, it is possible to 
calculate the melting temperature according to eq (5) and to compare it with the experimental data. As we can see from Fig. 14, the calculated curve 1 is situated approximately 400 degrees higher in the temperature scale, than the obtained points. Closer to room temperature, there is strong deviation from calculations even taking into account the above mentioned shift by 400 degrees (curve 2).

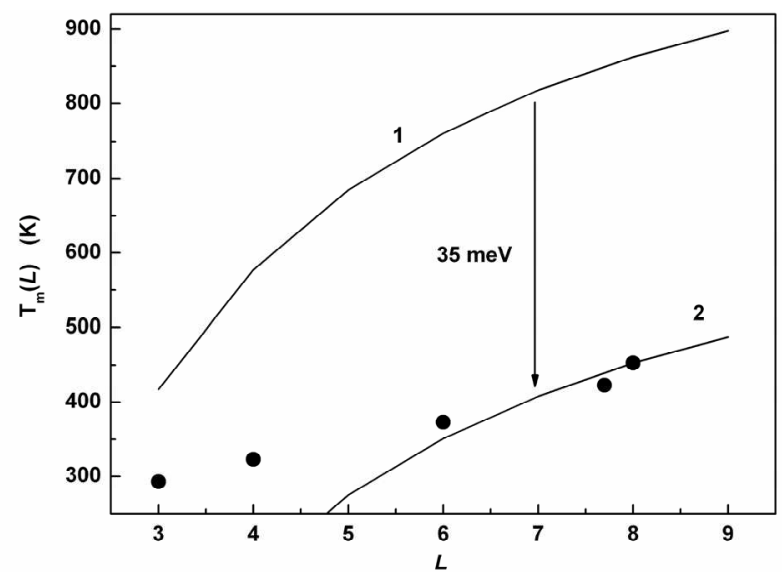

Fig. 19. Dependence between cluster melting temperature and its radius, curve 1 calculation, 2 - the same dependence shifted in energy by $35 \mathrm{meV}$, black circles experimental points.

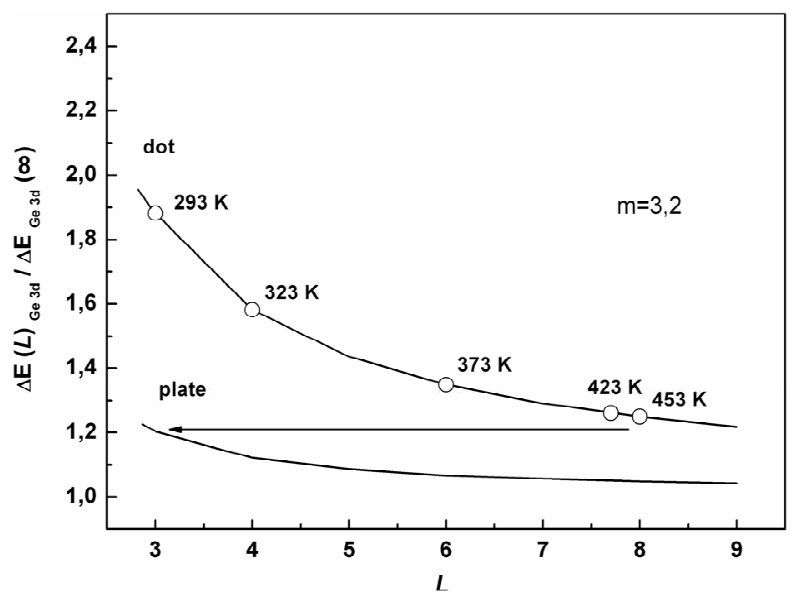

Fig. 20. Calculated dependence of the value of relative shift $\left[E_{3 d}(L)-E_{3 d}(1)\right] / \Delta E_{3 d}(\infty)$ of $G e$ $3 \mathrm{~d}$ level for cluster (continuous curve) and continuous layer (bottom dashed curve) on characteristic $L$ parameter: radius for a cluster and thickness for a layer. Transition to continuous monocrystal layer during recrystallization is shown with an arrow; specimen temperature is shown over the experimental points. 
Such discrepancy needs further explanation. In a previous work (Goldstein, 1995), the method of transmission electron microscopy allowed to observe in situ the melting process of Si nanoclusters, prepared according to a special procedure on a thin aluminium foil. Direct observation of changes in cluster forms and their crystal structure at different specimen temperatures via electronic microscope, made it possible to determine their melting temperature. It turned out, that melting temperature of $\mathrm{Si}$ clusters about $3 \mathrm{~nm}$ in diameter was in the range between 50 and $100{ }^{\circ} \mathrm{C}$. This value is lower than that calculated using BOLS model by about $700^{\circ}$ (Sun et al., 2001). Experimental data are once again significantly understated in comparison with theoretical predictions. Presumably, the observed deviations in both cases may be explained by physical influence of the "interrogating" radiation. It can be assumed, that part of X-ray quantum energy during external photoemission is spent not only on electron emission, but also to excite crystal lattice vibrations (optical phonon energy is $35 \mathrm{meV}$ in $\mathrm{Ge}$ and $63 \mathrm{meV}$ in $\mathrm{Si}$ ). Such additional heating of the cluster will reduce the observed melting temperature, and deviations will be the higher the smaller the cluster size and the higher the phonon energy are.

In the same work (Goldstein, 1995) coalescence of clusters and absorption of smaller clusters by larger ones was observed during melting. Similar processes should be taking place in our case. Using the described collection of experimental data, the recrystallization of a nanoscale Ge layer may be conceived as follows. With a rise in specimen temperature the influence of the electron (RHEED) or X-ray (XPS) beam causes melting of the smallest clusters, accompanied by their coalescence and enlargement. This process is favoured by a weak energy of Ge bond, with the epitaxial ZnSe layer and respectively low activation energy of adatom diffusion on the surface. After reaching a temperature about $620 \mathrm{~K}$, a continuous epitaxial intermediate Ge layer is formed, in which theoretically there could be Ge clusters with a higher melting temperature than the specified one. This sequence of events is shown in Fig. 22, and is indirectly confirmed by Ge 3d peak dispersion behaviour with temperature. Decrease in peaks dispersion in Fig. 2 indicates increase of the system homogeneity.

Thus, using the XPS method it is possible to observe fairly delicate effects related to a change of physical properties of the material on nanolevel. At the same time, it must always be kept in mind that any measurements are always accompanied with interaction between the device and the object, which introduces a certain error into the research results.

\section{Epitaxial layers of $\mathrm{CaF}_{2}$ and $\mathrm{BaF}_{2}$ on $\mathrm{Si}$}

Epitaxial films of fluorides of alkaline-earth metals with fluorite structure are extensively studied because of their dielectric properties, allowing their application as isolating layers in various electronic structures. For example, such materials as $\mathrm{CaF}_{2}$ and $\mathrm{BaF}_{2}$ are used as isolating buffer on $\mathrm{Si}$ for subsequent epitaxy of structures with quantum dots and solid solutions of lead chalcogenides. In the latter case, it is particularly topical in production of monolithic photodetectors using silicon technology. In (Hirose et al., 1992) the problem is studied of using $\mathrm{CaF}_{2}-\mathrm{SrF}_{2}$ fluorides as an electronic monocrystal resist on GaAs. That was possible because the influence of electronic beam causes breakdown of the compound accompanied by evaporation of fluorine. Subsequent metal oxidation and its dissolution make it possible to form the required pattern, and to preserve epitaxial isolating layer in other parts of the structure. 
In our work, we studied the mechanism of decomposition of $\mathrm{CaF}_{2}$ and $\mathrm{BaF}_{2}$ layers during the surface excitation with a diffractometer electron beam during MBE on Si substrate (Suprun \& Shcheglov, 2008). It was shown, that such influence causes formation of such macrodefects as pores. The degree of their development depends on doze, i.e. it is defined by the amount of received charge. It is accompanied by dissociation of the material under the influence of high-energy electrons, fluorine desorption, and transport of positive metal ions from the electron charge drain area. This phenomenon must be considered to exclude formation of defects in the working area when trying to obtain isolating layers. Moreover, this effect can be used in electronic lithography excluding contact between the produced structure and the atmosphere on intermediate stages. In this case, epitaxy combined with electronic scanning will allow immediate formation of the required pattern on the substrate, and preservation of epitaxial protective dielectric in other places. The next step could involve growth of the required layer in preliminary irradiated areas, doping, etc. without specimen exposing to the atmosphere.

$\mathrm{Si}-\mathrm{CaF}_{2}$ and $-\mathrm{BaF}_{2}$ heterosystems have a few more peculiarities, related to the interface formation and which have not been satisfactory explained so far. It is clear, that some defects - stoichiometry irregularities, formed in the zone of transition from crystal lattice of one material to another, become electrically active centres. They can develop during observation of different electrophysical characteristics. The statement looks quite obvious: the more defects, the worse. More specifically, the greater is the mismatch in lattice constant in the heterojunction, the higher is the density of electrically active states in the interface. Let us consider an example which contradicts such simplified approach to the problems related to physical and chemical processes of heterointerface formation and its electronic properties.

$\mathrm{Si}-\mathrm{CaF}_{2}$ heterosystem has been quite extensively studied, which is true both for its formation using the MBE method and its electrophysical properties. Existing literature describes results of studying the behaviour of capacitance-voltage $\mathrm{C}-\mathrm{V}$ characteristics of MISstructures with $\mathrm{CaF}_{2}$ films grown at different temperatures (Cho et al., 1992). It was shown, that the value of capacity modulation of the obtained structures depends on the temperature of $\mathrm{CaF}_{2}$ deposition. The higher was the substrate temperature during the growth of epitaxial layers in the interval $(300-700)^{\circ} \mathrm{C}$, the narrower was the range of capacity change from voltage, and no modulation at all was observed in specimens obtained at $\mathrm{T} \approx 700^{\circ} \mathrm{C}$. Analysis of electrophysical data and XPS results allowed authors of (Ioannov-Sougleridis et al., 1998; Olmstead et al., 1986) to draw a conclusion, that the reasons of the observed effect are physical and chemical changes in $\mathrm{Si}_{-}-\mathrm{CaF}_{2}$ heterointerface. Partial dissociation of $\mathrm{CaF}_{2}$ molecules occurs during deposition, and the intensity of the process grows with a rise in the substrate temperature. This is accompanied by a reduction of Si-F bonds density at the interface, which can be observed as decrease in the intensity of high-energy component of $\mathrm{F}$ $2 \mathrm{p}$ doublet in XPS spectra. Thus, different types of bonds can be formed at the heterointerface - Si-F ionic type bonds, mainly at low temperature, and relatively weaker metal bond $\mathrm{Si}-\mathrm{Ca}$ at high temperature and lack of fluorine. The authors of the aforementioned works offered a detailed analysis of Si $2 p$ peak components. It was shown, that its chemical shift by $0.83 \mathrm{eV}$ towards higher binding energies in relation to bulk component (Si-Si bond) takes place during formation of Si-F bond, and shift by $0.36 \mathrm{eV}$ towards lower binding energies occurs in the presence of bond with calcium. 
The results of our experiments (Suprun \& Shcheglov, 2008) with $\mathrm{Si}_{-} \mathrm{CaF}_{2}$ system are in good agreement with other well-known data. Besides, we noted improvement in layers adhesion with a decline in deposition temperature, which is consistent with the assumption that predominant type of bonds in the heterointerface zone changes with temperature. As for Si$\mathrm{BaF}_{2}$ heterosystem, there is a rather small number of works devoted to its analysis. This is caused, first of all, by a very large mismatch in lattice constants of these materials $\approx 14 \%$ (for comparison $\mathrm{Si}-\mathrm{CaF}_{2}-\approx 0.6 \%$ ), therefore it seems that there might be problems to obtain good quality interface in this case. Authors of (Belenchuk et al., 2000) analyzed structure and morphology of the surface of $\mathrm{BaF}_{2}$ films grown with the help of $\mathrm{MBE}$ method on $\mathrm{Si}$ substrates (001). One of the growth peculiarities here is the formation of an oriented $\mathrm{BaF}_{2}$ layer (111), which means that there is a change of crystallographic orientation in relation to the substrate. The atomic force microscopy AFM showed the presence of well-developed block borders several microns in size. Electrophysical properties of such structures were never studied. According to our estimations, the density of dangling bonds at the interface from the side of $\mathrm{Si}$, having a smaller lattice constant, will reach values of $3 \times 10^{14} \mathrm{~cm}^{-2}$, and mismatch dislocations form a grid with a step about $3 \mathrm{~nm}$.

The results of our experiments, studying the system $\mathrm{Si}-\mathrm{BaF}_{2}$, allowed us to assume that $\mathrm{BaF}_{2}$ films on silicon were grown in MBE installation according to the procedure described in (Suprun \& Shcheglov, 2008). We used a thermal evaporator as a $\mathrm{BaF}_{2}$ source, loaded with nutrient prepared from a monocrystal. Deposition rate was $2-6 \mathrm{~nm}$ per minute. Thickness of the obtained layers was in the range of $(0.2$ - 0.4) micron. We used Si (001) plates $76 \mathrm{~mm}$ in diameter as substrates, initially covered with oxide and undergoing standard chemical preparation before they were loaded into the installation. The substrates were cleaned by heating in vacuum at $850^{\circ} \mathrm{C}$ until the appearance of the surface superstructure $7 \times 7$, then $\mathrm{BaF}_{2}$ deposition began at temperature of $(750-780)^{\circ} \mathrm{C}$. Diffraction picture in the process of epitaxy corresponded to formation of a block monocrystal. To protect the surface of the obtained film from the atmosphere influence, in some cases we used $\mathrm{CaF}_{2}$ deposition at $700^{\circ} \mathrm{C}$.

To study the chemical structure of the interface, with the help of XPS method, a layer of $\mathrm{BaF}_{2}$ $\approx 1 \mathrm{~nm}$ thick was grown at a substrate temperature $780^{\circ} \mathrm{C}$. The spectra of $\mathrm{Si}-\mathrm{BaF}_{2}$ specimen were obtained using $\mathrm{Al} \mathrm{K}$ line as the source of radiation; spectra were acquired with $0.6 \mathrm{eV}$ resolution with analyzer inclination $20^{\circ}$ and $70^{\circ}$ off the normal to the sample surface in order to vary the effective depth of analysis.

Fig. 23 shows Si 2 p peak. Table 1 below shows the spin-orbital splitting values of this peak (components ratio in the doublet is 2:1), as well as values of full width at half-height of Gauss and Lorentz functions used in experimental spectra decomposition, energy shifts for surface component of silicon, and its compounds in relation to its bulk component. The values shown in the table 3 were taken from (Olmstead et al., 1986). The shift value for Si-Ba bond was determined in the course of spectrum decomposition, and slightly differed from similar energy shift for Si-Ca bond.

Si 2 p spectra in Fig. 23 (energy position of bulk component $V-99.01 \mathrm{eV}$ ), were acquired at specimen inclination angle 20 (above) and 70 degrees to the normal. Relative contribution of each component (according to the area under the peak) is shown in table 4.Experimental data are shown in fig. 23 by circles, dashed lines corresponding to decomposition 
components, continuous line being the sum of all components. Designations on the spectra: $V$ is a component, corresponding to $\mathrm{Si}$ bulk bond, $S$ is a surface component, $\mathrm{Si}$ (Ba) is siliconbarium bond. Note, that presence of fluorine in the heterointerface area is within the error of the fitting procedure for the initial experimental spectrum. Comparison of peaks in the top and bottom Fig. 23 indicates that $\mathrm{Si}-\mathrm{BaF}_{2}$ interface is formed owing to $\mathrm{Si}-\mathrm{Ba}$ bond: intensity of this component sharply increases with reduction of the effective depth of analysis. This conclusion is consistent with a type of spectrum corresponding to F $2 \mathrm{p}$ doublet (similar to the one described in (Olmstead et al., 1986)), where the intensity of high-energy components is smaller, indicating a lack of fluorine in the interface zone.

\begin{tabular}{|l|l|}
\hline Si $2 \mathrm{p}$ & Energy $[\mathrm{eV}]$ \\
\hline Spin-orbital splitting $(\mathrm{eV})$ & 0.600 \\
\hline Gaussian width $(\mathrm{eV})$ & 0.6 \\
\hline Lorentzian width $(\mathrm{eV})$ & 0.7 \\
\hline Surface component S (eV) & 0.44 \\
\hline Chemical shift- Si $(\mathrm{eV})$ in bond & \\
\hline Si-F & 0.83 \\
\hline Si-Ba & -0.35 \\
\hline Si-Ca & -0.36 \\
\hline
\end{tabular}

Table 3.

\begin{tabular}{|l|l|l|}
\hline Component & Sk $/ \mathrm{S}_{0}\left(20^{\circ}\right)$ & Sk $/ \mathrm{S}_{0}\left(70^{\circ}\right)$ \\
\hline Bulk $(\mathrm{V})$ & 0.874 & 0.393 \\
\hline Si-Ba & 0.099 & 0.603 \\
\hline Surface component S & 0.026 & 0.003 \\
\hline Si-F & 0.001 & 0.001 \\
\hline
\end{tabular}

Table 4.

Thus, high-temperature growth of both calcium fluoride, according to the data in the research literature, and barium fluoride (in our experiments) results in the predominant formation of silicon bond with alkaline-earth metal in the interface zone. Of particular interest are the obtained measurement results of the electrophysical characteristics of $\mathrm{Si}-\mathrm{BaF}_{2}$ specimens. It is known, that no capacity modulation was observed in $\mathrm{Si}_{-} \mathrm{CaF}_{2} \mathrm{MIS}$-structures obtained at temperature $700^{\circ} \mathrm{C}$; moreover, the mismatch in lattice constant for $\mathrm{Si}-\mathrm{BaF}_{2}$ system is more than 10 times higher. Judging by the conventional opinion, there can hardly be something good to expect in this situation.

We performed measurements of $\mathrm{C}-\mathrm{V}$ and $\mathrm{G}-\mathrm{V}$ characteristics using immitance bridge with error in capacity measurement $\pm 0.1 \%$, and of parallel conductivity with error $\pm 1 \%$. Two types of contacts were used in specimens measurement: mercury contact $S=1.610^{-3} \mathrm{~cm}^{2}$ by area, and mask deposited in vacuum contact $(\mathrm{Ti}+\mathrm{Ag}) \mathrm{S}=510-3 \mathrm{~cm}^{2}$ in area. Properties of these structures were measured at frequencies $1.66 \mathrm{kHz}, 16.6 \mathrm{kHz}$ and $100 \mathrm{kHz}$.

Before the formation of a metal electrode with the help of LEF 3 ellipsometer with operating wave length of laser radiation 0.63 micron, we defined refraction index $(n)$ and thickness $(d)$ 
of the $\mathrm{BaF}_{2}$ layer. The spread in refraction index values at different points on the surface was in the range from 1.465 to 1.473, and the spread in layer thickness was from $373 \mathrm{~nm}$ to 388 $\mathrm{nm}$. According to reference data (Grigorjeva, 1991) at wavelength of 0.5893 microns refraction index of $\mathrm{BaF}_{2}$ volume material is 1.4775, and at wavelength of 1 micron it equals 1.4686.Thus, the refraction index of deposited films is consistent with the previously known results. Electric strength of the layers was measured at let-through current $10-5 \mathrm{~A}$, and was in the range of $(0.6-1.5) \times 10^{6} \mathrm{~V} \times \mathrm{cm}^{-1}$.

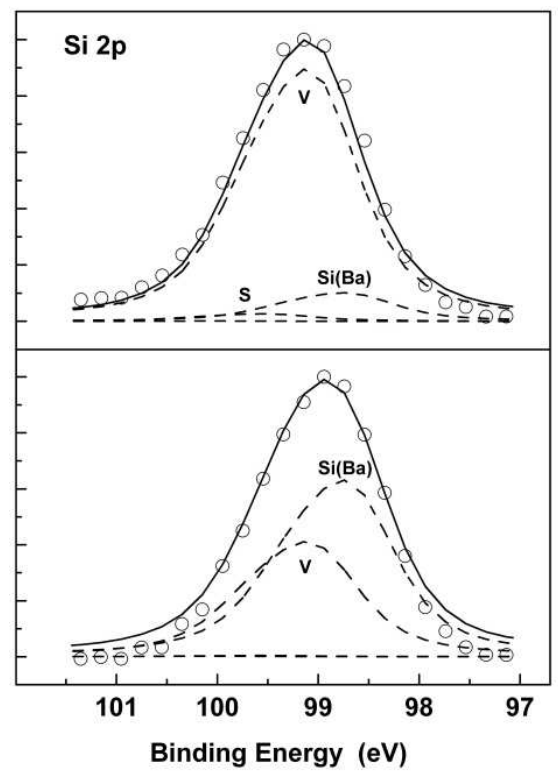

Fig. 21. Si 2 p spectra acquired for the specimen inclination angle $20^{\circ}$ (above) and $70^{\circ}$ (below) to the normal. Components designations are described in the text.

Fig. 24 and 25 show the results of our analysis of $\mathrm{Si}(001)-\mathrm{BaF}_{2}$ specimens (substrate temperature during $\mathrm{BaF}_{2}$ growth was $780^{\circ} \mathrm{C}$ ) and $\mathrm{Si}(001)-\mathrm{BaF}_{2}-\mathrm{CaF}_{2}$ (substrate temperature during $\mathrm{BaF}_{2}$ growth was $780^{\circ} \mathrm{C}$, temperature during $\mathrm{CaF}_{2}$ deposition was $700^{\circ} \mathrm{C}$ and its thickness was $40 \mathrm{~nm}$ ). High-resistivity silicon with n-type conductivity was used as a substrate.

The characteristics shown were measured during the change of the polarizing voltage from a strong inversion mode of MIS-structures to a strong enhancement mode in the range $(-15$; $+15) \mathrm{V}$. In the enhancement mode the capacity of MIS- structure, as wellknown, equals the dielectric layer capacity $\left(C_{d}\right)$. Using the ellipsometric measurements data of the thickness of $\mathrm{BaF}_{2}$ layer and the measured value $C_{d}$ with the known area of metal contact $S$, we obtained the dielectric constant for grown $\mathrm{BaF}_{2}$ layers.

Calculated value of $\varepsilon$ BaF2 $=6.9$ differs from the value $\varepsilon$ BaF2 $=7.4$, obtained in [7] for a monocrystal. The difference may be caused by an error in the estimation of the contact area, owing to considerable relief of the surface or peculiar properties of the film itself. 
Fig. 24 and 25 illustrate frequency dispersion of C-V dependences connected with states existing at the interface. These states are also characterized by the presence of maxima on $\mathrm{G} / \omega-\mathrm{V}$ dependences. Note, that no peak is observed on $\mathrm{G} / \omega-\mathrm{V}$ characteristics at the frequency of $100 \mathrm{kHz}$. It proves that density of the states at the interface with the time constant $\tau$, corresponding to that frequency and with smaller values is very low. Thus, $\mathrm{C}-\mathrm{V}$ characteristics for the studied specimens, measured at $100 \mathrm{kHz}$ can be considered as highfrequency.

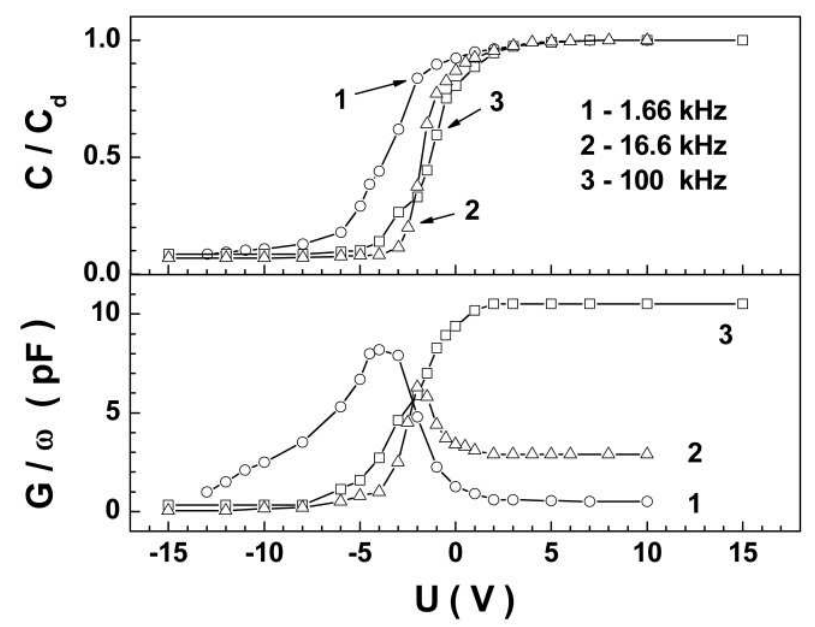

Fig. 22. $\mathrm{C}-\mathrm{V}$ (top) and G-V (bottom) characteristics of $\mathrm{Si}(001)-\mathrm{BaF}_{2}-\mathrm{Hg}$ structures, measured for three different frequency values.

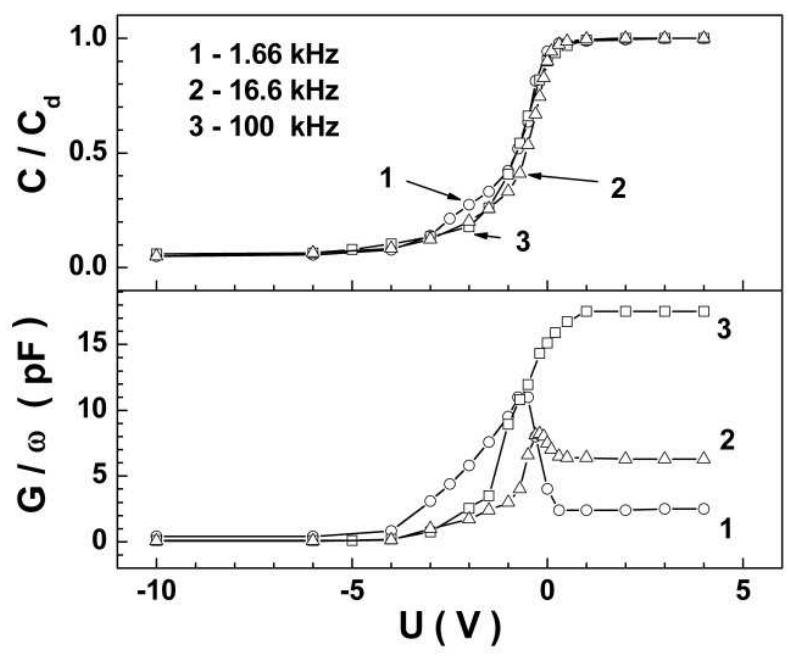

Fig. 23. $\mathrm{C}-\mathrm{V}$ (top) and G-V (bottom) characteristics of $\mathrm{Si}(001)-\mathrm{BaF}_{2}-\mathrm{CaF}_{2}-(\mathrm{Ti}+\mathrm{Ag})$ structures, measured for three different frequency values. 
From $\mathrm{C}-\mathrm{V}$ and $\mathrm{C}-\mathrm{V}$ и $\mathrm{G} / \omega-\mathrm{V}$ characteristics we obtained the density of states $\mathrm{N}_{\mathrm{SS}}$ at $\mathrm{Si} / \mathrm{BaF}_{2}$ interface. The calculated values of density of state for $\mathrm{Si}(001) / \mathrm{BaF}_{2}$ specimen are as follows: at $\tau=100 \mu \mathrm{sec}-N_{\mathrm{SS}}=1.84 \times 10^{11} \mathrm{eV}^{-1} \times \mathrm{cm}^{-2}$, at at $\tau=10 \mu \mathrm{sec}-N_{\mathrm{SS}}=9.8 \times 10^{10} \mathrm{eV}^{-1} \times \mathrm{cm}^{-2}$. For the second specimen at $\tau=100 \mu \mathrm{sec}-N_{\mathrm{SS}}=1.1 \times 10^{11} \mathrm{eV}^{-1} \times \mathrm{cm}^{-2}$, and at $\tau=10 \mu \mathrm{sec}-N_{\mathrm{SS}}=$ $1.07 \times 10^{11} \mathrm{eV}^{-1} \times \mathrm{cm}^{-2}$.

The experimental data given above bring to the conclusion, that the capacitance modulation of $\mathrm{Si}-\mathrm{BaF}_{2}$ structure is observed if the predominant bond in the heterointerface is $\mathrm{Si}-\mathrm{Ba}$. This case essentially differs from the results obtained for $\mathrm{Si}_{-} \mathrm{CaF}_{2}$ system. Density of dangling bonds at the interface $\mathrm{Si}-\mathrm{BaF}_{2}$, calculated from the difference between lattice constants approximately by three orders of magnitude, exceeds the density of states obtained experimentally on the basis of electrophysical characteristics. Analysis of the interface with the help of XPS method, and the method used to obtain the structure guarantees the absence of any foreign elements which could neutralize dangling Si bonds. It is safe to assume, that high density of spatially ordered defects itself forms a quantum subsystem, but this problem requires additional studies.

\section{Conclusion}

XPS technique allows to study various processes on a level of the electronic shell structure. Such approach breaks the familiar sight of many concepts, so we can see the "virtual" reference point, which relates to the energy of a pure isolated atom. We seldom realize that in experiments we only can observe reliably transitions between various chemical states of materials. In such transitions, for example, at the boundary between two stoichiometric monocrystals, such a macro-level characteristic as composition is determined by matching dipole moments, i.e. by a condition that has to be met on a micro-level. Application of XPS method also brings to the conclusion that obtaining any experimental data is unavoidably accompanied by an interaction with the analyzed object, which changes its energy state.

To summarize, it is necessary to point out that the discussed experimental results, obtained on many diverse heterostructures, show that XPS in situ technique can be effectively applied to study various phenomena at interfaces and chemical reactions in solid phase.

\section{Acknowledgments}

The work was supported by the Russian Foundation for Basic Research under Project No. 11-07-12067. The authors wish to thank V.G. Erkov for providing data on electrophysical properties of $\mathrm{Si}--\mathrm{BaF}_{2}$ MIS-structures.

\section{References}

Ahrenkel, R.K.; Kazmerski, L.L.; Ireland, P.J.; Jamjoum, O.; Russell, P.E.; Dunlavy, D.; Wagner, R.S.; Pattillo, S. \& Jervis, T. (1982). Reduction of surface states on GaAs by the plasma growth of oxyfluorides. J. Vac. Sci. Technol., Vol. 21(2), pp. 434-437, ISSN 0022-5355

Bauer, R.S.; (1983). The role of metastable surfaces in determining MBE heterojunction structure: GaAs/Ge interfaces. J. Vac. Sci, Technol. B, 1, p. 314, ISSN 0734-211 
Belenchuk, A.; Fedorov A., Huhtinen H., Kantser V., Laiho R., Shapoval O. \& Zakhvalinskii V., (2000). Growth of (111)-oriented PbTe films on $\mathrm{Si}(001)$ using a BaF2 buffer Thin Solid Films, pp. 358, 277-282. ISSN 0040-6090

Briggs, D. \& Seah M.P. (Eds.) (1990). Auger and X-ray Photoelectron Spectroscopy, Practical Surface Analysis, Vol. 1, ISBN-13: 978-0471953401, Wiley, Chichester

Carli, R. \& Bianchi C.L. (1994). XPS analysis of gallium oxides. Appl. Sur. Sci., 74, 1, pp. 99102, ISSN 0169-4332

Chang, C.C.; Chang, R.P.H. \& Murarka, S.P. (1978). Plasma-grown oxide on GaAs. J. Electrochem. Soc., Vol. 125(3), pp. 481-487, ISSN 0013-4651

Cho, C.-C. ; Kim T. S., Gnade B. E., Liu H. \& Y. Nishioka Y. (1992). Electrical properties of low-temperature-grown $\mathrm{CaF}_{2}$ on Si(111). Appl. Phys. Lett., 60, pp. 338-340, ISSN 0003-6951

Davey, J.E.; Tierman R.J., \& Pankey T. \& Montgomery M.D. (1963). The effect of vacuumevaporation parameters on the structural, electrical and optical properties of thin germanium films. Solid-State Electr., 6, 3, 205-212, ISSN 0038-1101

Dultsev, F.N. \& Kesler, V.G. (2009). Etching and oxidation of InAs in a planar inductively coupled plasma. Appl. Surf. Sci., Vol. 256, pp. 246-250, ISSN 0169-4332

Franciosi, A. \& Van de Walle C.G. (1996). Heterojunction band offset engineering. Surf. Sci. Reports, 25, pp.1-140, ISSN 0167-5729

Gleim, Th.; Heske C., Umbach E., Schumacher C., Gundel S., Faschinger W., Fleszar A., Ammon Ch., Probst M., \& Steinruck H.-P. (2003). Formation of the ZnSe/(Te/)GaAs(100) heterojunction. Sur. Sci., 531, 77-85, ISSN 00396028

Goldstein, A.N. ; (1995). The melting of silicon nanocrystals: Submicron thin-film structures derived from nanocrystal precursors. Appl. Phys. A 62, 1, pp. 33-37, ISSN 0947-8396

Grigorjeva, I.S. \& Mejlihov E.Z. (Eds.), (1991). Fizicheskie velichiny. Energoatomizdat, ISBN: 5283-04013-5, Moskva

Grigorovich, S.L.; Volkov, A.S. \& Lotsman, A.P. (1981). Study of the structure of anode oxide films based on indium arsenide using the method of Auger electron spectroscopy. Electronic technology. Serial materials, Issue 3 (152), pp. 39-42, ISSN

Grunthaner, P.J.; Grunthaner F.J., Fathauer R.W., Lin T.L., Hecht M.H., Bell L.D., Kaiser W.J., Schowengerdt F.D. \& Mazur J.H. (1989). Hydrogen-terminated silicon substrates for low-temperature molecular beam epitaxy. J.Thin Solid Films, 183, pp. 197-212, ISSN 0040-6090

Gurtov, V.A.; Zolotov, M.V.; Kovchavtsev, A.P. \& Kuryshev, G.L. (1986). Volume charge in MIS-structures based on indium arsenide. Microelectronics, Volume 15, issue 2, pp. 142-145, ISSN 0544-1269

Hirose, Y. ; Horng S., Kahn A., Wrenn C. \& Pfeffer R. (1992). Electron beam patterning of epitaxial $\mathrm{CaF}_{2}$ and $\mathrm{Ca}_{0.5} \mathrm{Sr}_{0.5} \mathrm{~F}_{2}$ /(100)GaAs J.Vac.Sci.Technol. A 10, 4, pp. 960-964, ISSN 0734-2101

Holloway, P. H. \& McGuire G.E. (Eds.), (1995). Handbook of Compound Semiconductors, William Andrew Inc., New Jersey, ISBN 0-8155-1374-7

Howard, A. ; Clark D.N.S., Mitchell C.E.J., Egdell R.G. \& Dhanak V.R. (2002). Initial and final state effects in photoemission from Au nanoclusters on TiO2(110). Surf. Sci. 518, 3, pp. 210-224, ISSN 00396028 
Ioannov-Sougleridis, V. ; Tsakiri V., Nassiopoulou A. G., Photopoulos P., Bassani F. \& d' Avitaya F.A. (1998). Electroluminescence from Si/CaF2 Multilayers Grown by Molecular Beam Epitaxy. Phis. Stat. Sol. (a) 165, pp. 97-103,. ISSN 18626319

Isomura, N. ; Tsukamoto S., Iizuka K. \& Arakawa Y. (2007). Investigation on GaAs(001) surface treated by As-free high temperature surface cleaning method. J. Cryst. Growth, 301-302, pp. 26-29, ISSN 0022-0248

Kawanaka, M. \& Sone J. (1990). Current-voltage characteristics of p-Ge/n-GaAs heterojunction diodes grown by molecular beam epitaxy. J. Electronic Materials, 19, 6, pp. 575-580, ISSN 0361-5235

Kesler, V.G.; Seleznev, V.A.; Kovchavtsev, A.P. \& Guzev, A.A. (2010). Composition, morphology and surface recombination rate of $\mathrm{HCl}$-isopropanol treated and vacuum annealed InAs(111)A surfaces. Appl. Surf. Sci., Vol. 256, pp. 4626-4632, ISSN 0169-4332

Kesler, V.G.; Kovchavtsev, A.P.; Guzev, A.A.; Panova, Z.V. \& Kuryshev, G.L. (2010a). Anodic oxidation of indium arsenide in glow discharge oxygen plasma. Fundamental problems of modern materials engineering, Vol. 7, No. 4, pp. 82-88, ISSN 1811-1416

Kesler, V.G. (2011). Study of InAs oxidation process in glow discharge plasma using the XPS method in situ. Journal of structural chemistry, 52, , No. 8, pp. S156-S164, ISSN 0136-7463

Kornyushkin, N.A.; Valisheva, N.A.; Kovchavtsev, A.P. \& Kuryshev, G.L. (1996). Influence of properties of the interface and deep levels in low-energy gap on voltagecapacitance characteristic of MIS-structures on indium arsenide. FTP, Vol.30, issue 5, pp. 914-917, ISSN 0015-3222

Kovchavtsev, A.P.; Kuryshev, G. L.; Kanter, Yu.O.; Olshanetsky, B.Z.; Demyanov E.A.; Krytsyn, S.M. \& Stenin, S.I. (1986). Influence of the composition of transitive layer on the value of low-voltage hysteresis of voltage-capacitance characteristics of MISstructures based on indium arsenide. Surface. Physics, chemistry, mechanics, Vol. 10, pp. 132-137, ISSN

Kovchavtsev, A.P. et al. (2001). Photodetectors with charge injection on indium arsenide. In: Matrix photodetectors of infra-red range, V.N.Ovsyuk, G.L.Kuryshev, J.G.Sidorov et al., pp. 10-113, Nauka, ISBN 5-02-031717-9, Novosibirsk.

Lay, G.L. ; Mao D., Kahn A. \& Hwu Y. (1991). Margaritondo G. High-resolution synchrotron-radiation core-level spectroscopy of decapped GaAs(100) surfaces. Phys. Rev. B, 43, pp. 14301-14304, ISSN 1098-0121

Leandersson, S., K.; Gothelid, M.; Tjernberg, O. \& Karlsson, U.O. (2003). Interaction between oxygen and InAs(111) surfaces, influence of the electron accumulation layer. Appl. Surf. Sci, Vol. 212-213, pp. 589-594, ISSN 0169-4332

Li N., Harmon, E. S.; Hyland, James; Salzman, David B.; Ma, T. P.; Xuan, Yi \& Ye, P.D. (2008). Properties of InAs metal-oxide-semiconductor structures with atomiclayer-deposited $\mathrm{Al}_{2} \mathrm{O}_{3}$ dielectric. Appl. Phys. Lett., Vol. 92, p. 143507, ISSN 00036951 
Monch, W. \& Grant H. (1980). Combined LEED, AES, and work function studies during the formation of Ge : GaAs(110) heterostructures. J.Vac. Sci, Technol. 17, 5, pp. 10941100, ISSN 0022-5355

Nanda, J. ; Kuruvilla B.A. \& Sarma D.D. (1999). Photoelectron spectroscopic study of CdS nanocrystallites. Phys. Rev. B 59, pp. 7473-7479, ISSN 1098-0121

Neizvestny, I.G. ; Suprun S.P., Shumsky V.N., Talochkin A.B., Fedosenko E.V., Burbaev T.M. \& Kurbatov V.A. (2001). Quantum dots of Ge in a GaAs/ZnSe/Ge unstrained heterosystem: fabrication and properties. Nanotech. 12, 12, pp. 437-440, ISSN 09574484

Olmstead, M.A. ; Uhrberg I.G., Bringans R.D. \& Bachrach R.G. (1986). Photoemission study of bonding at the $\mathrm{CaF}_{2}$-on-Si(111) interface. Phys. Rev. B 35, pp.7526-7532, ISSN 1098-0121

Pan, L.K. ; Sun C.Q., Tay B.K., Chen T.P. \& Li S. (2002). Photoluminescence of Si Nanosolids near the Lower End of the Size Limit. J. Phys. Chem. B, 106, 45, pp. 11725-11727, ISSN 1089-5647

Pashley, M.D. ; (1989). Electron counting model and its application to island structures on molecular-beam epitaxy grown GaAs(001) and ZnSe(001). Phys. Rev. B, 40, 1048110487, ISSN 1098-0121

Schmeißer, D. ; Böhme O., Yfantis A., Heller T., Batchelor D.R., Lundstrom I. \& Spetz A.L. (1999). Dipole Moment of Nanoparticles at Interfaces. Phys. Rev. Lett. 83, pp. 380383, ISSN 0031-9007

Schwartz, R.J.; Dockerty, R.C. \& Thompson, H.W. (1971). Capacitance voltage measurements on n-type InAs MOS diodes. Sol. St. Electron., Vol.14, pp. 115-124, ISSN 0038-1101

Seah, M.P., Gilmore I.S. \& Spencer S.J. (2001). Quantitative XPS. Journal of Electron Spectroscopy and Related Phenomena, 120, pp. 93-111. ISSN: 0368-2048

Seah, M.P. \& Spenser, S.J. (2002). Ultrathin $\mathrm{SiO}_{2}$ on Si. II Issues in quantification of the oxide thickness. Surf. Interface Anal., Vol. 33, p. 640-652, ISSN 0142-2421

Sorokin, I.N. \& Gatko, L.E. (1985). Influence of fluorine on growth and properties of anode oxide layers of indium arsenide. Inorganic materials, Vol. 21, No. 4, pp. 537-540, ISSN 0002-337X

Stucki, F., Lapeyre G.J., Bauer R.S., Zurcher P. \& Mikkelsen J.C., Jr. (1983). Photoemission study of GeAs(01): A model for the As-stabilized Ge surface on GaAs/Ge heterojunctions. J. Vac. Sci, Technol. B, 1, pp. 865-870, ISSN 0734-211

Sun, C. Q. (2007). Size dependence of nanostructures: Impact of bond order deficiency. Progress in Solid State Chemistry, 35, pp. 1-159, ISSN: 0079-6786

Sun, Y.; Pianetta Po-Ta Chen, P.; Kobayashi, M.; Nishi, Y.; Goel, N.; Garner, M. \& Tsai, W. (2008). Arsenic-dominated chemistry in the acid cleaning of InGaAs and InAlAs surfaces. Appl. Phys. Lett., Vol. 93, p. 194103, ISSN 0003-6951

Sun, C.Q. ; (2004). Surface and nanosolid core-level shift: Impact of atomic coordinationnumber imperfection. Phys. Rev. B 69, 045105, ISSN 1098-0121

Sun, C.Q. ; Chen T.P., Tay B.K., Li S., Huang H., Zhang Y.B., Pan L.K., Lau S.P. \& Sun X.W. (2001). An extended 'quantum confinement' theory: surface-coordination imperfection modifies the entire band structure of a nanosolid. J. Phys. D: Appl. Phys. 34, 24, pp. 3470-3479, ISSN 0022-3727 
Suprun, S.P.; Gutakovskiy A.K. Torlin M.A., Fedosenko E.V., Sherstyakova V.N., \& Shumsky V.N. (1995). Structural and electrophysical properties of PtSi_Si the Shottky barriers J.Phys. Low-Dim. Struct., 10/11, pp. 125-132, ISSN 0204-3467

Suprun, S.P.; Talochkin A.B., Gutakovskiy A.K. \& Shumsky V.N. (1995a). Determination Of Thickness Of Heteroepitaxial Ultra Thin Layers By RAMAN Spectroscopy. J.Phys. Low-Dim. Struct., 1, pp. 59-66, ISSN 0204-3467

Suprun, S.P. ; Torlin M.A., Fedosenko E.V., Sherstyakova V.N., Shumsky V.N. (1997). Characterization of surface beam roughness during molecular epitaxy on GaAs substrates by mesurements of scattered laser radiation intensity, pp. 119-122, Proceedings of ISDRS-97, Sharlottesville, USA, December

Suprun, S.P. \& Fedosenko E.V. (2009). Formation of the GaAs-Ge heterointerface in the presence of oxide. JETP Letters, 89, 2, pp. 94-97, ISSN 0044-4510

Suprun, S.P. \& Fedosenko E.V. (2007). Low-temperature recrystallization of Ge nanolayers on ZnSe. Semiconductors, 41, pp. 590-595, ISSN 1063-7826

Suprun, S.P. ; Sherstyakova V.N. \& Fedosenko E.V. (2009). Epitaxial growth of ZnSe on GaAs with the use of the ZnSe compound as the source. Semiconductors, 43, pp. 1526-1531, ISSN 1063-7826

Suprun, S. P. \& Shcheglov D. V. (2008). Effect of an Electron Beam on CaF2 and BaF2 Epitaxial Layers on Si. JETP Letters, Vol. 88, No. 6, pp. 365-369. ISSN 00444510

Talochkin, A.B. ; Teys S.A. \& Suprun S. P. (2005). Resonance Raman scattering by optical phonons in unstrained germanium quantum dots. Phys. Rev. B 72, pp. 115416, ISSN 1098-0121

Tereshchenko, O.E.; Alperovich, V.L. \& Terekhov, A.S. (2006). Composition and structure of chemically prepared GaAs(111)A and (111)B surfaces. Surf. Sci., Vol. 600, p. 577, ISSN 0039-6028

Tereshchenko, O.E.; D. Paget, P. Chiradia, J.E. Bonnet, F. Wiame, and A. Naleb-Ibrahimi, Preparation of clean reconstructed InAs(001) surfaces using $\mathrm{HCl}$ /isopropanol wet treatments, Appl. Phys. Lett. 82 (24) (2003) 4280

Tereshchenko, O.E.; Paget, D.; Chiradia, P.; Bonnet, J.E.; Wiame, F. \& Naleb-Ibrahimi, A. (2003). Preparation of clean reconstructed InAs(001) surfaces using $\mathrm{HCl}$ /isopropanol wet treatments. Appl. Phys. Lett., Vol. 82 (24), p. 4280, ISSN 00036951

Torkhov, N.A. ; (2003). Formation of a native-oxide structure on the surface of n-GaAs under natural oxidation in air. Semiconductors, 37, 10, pp. 1177-1184, ISSN 10637826

Valisheva, N.A.; Levtsova, T.A.; Logvinsky, L.M.; Kovchavtsev, A.P.; Kuryshev, G.L.; Petrenko, A.Z. \& Petrenko I.P. (1999). Influence of fluorine on the interface anode oxide-indium arsenide. Journal of Surface Investigation. X-Ray, Synchrotron and Neutron Techniques, No. 11, pp. 53-58, ISSN 0207-3528

Van Buuren, T. ; Weilmeier M.K., Athwal I., Colbow K.M., Mackenzie J.A., \& Tiedje T. (1991). Oxide thickness effect and surface roughening in the desorption of the oxide from GaAs. Appl. Phys. Lett., 59, 4, pp. 464-466, ISSN 0003-6951

Yamada, M. \& Ide Y. (1995). Anomalous behaviors observed in the isothermal desorption of GaAs surface oxides. Sur. Sci., 339, pp. L914-L918, ISSN 00396028 
Yu E.T., McCaldin J.O. \& McCaldin T.C., Band offset in semiconductor heterojunctions. Solid St. Phys., 46 (1992), pp. 1-146, ISSN 0-12-607746-0 


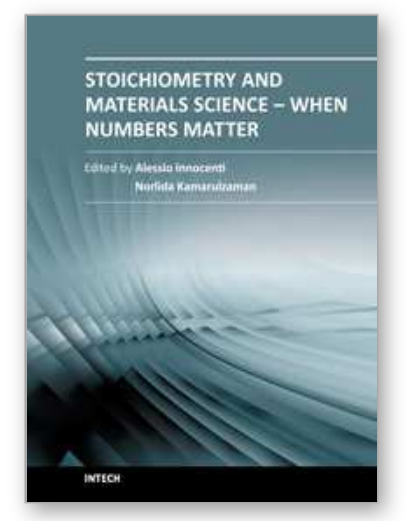

\author{
Stoichiometry and Materials Science - When Numbers Matter \\ Edited by Dr. Alessio Innocenti
}

ISBN 978-953-51-0512-1

Hard cover, 436 pages

Publisher InTech

Published online 11, April, 2012

Published in print edition April, 2012

The aim of this book is to provide an overview on the importance of stoichiometry in the materials science field. It presents a collection of selected research articles and reviews providing up-to-date information related to stoichiometry at various levels. Being materials science an interdisciplinary area, the book has been divided in multiple sections, each for a specific field of applications. The first two sections introduce the role of stoichiometry in nanotechnology and defect chemistry, providing examples of state-of-the-art technologies. Section three and four are focused on intermetallic compounds and metal oxides. Section five describes the importance of stoichiometry in electrochemical applications. In section six new strategies for solid phase synthesis are reported, while a cross sectional approach to the influence of stoichiometry in energy production is the topic of the last section. Though specifically addressed to readers with a background in physical science, I believe this book will be of interest to researchers working in materials science, engineering and technology.

\title{
How to reference
}

In order to correctly reference this scholarly work, feel free to copy and paste the following:

Sergey P. Suprun, Valeriy G. Kesler and Evgeniy V. Fedosenko (2012). Observation of Chemical Reactions in Solid Phase Using X-Ray Photoelectron Spectroscopy, Stoichiometry and Materials Science - When Numbers Matter, Dr. Alessio Innocenti (Ed.), ISBN: 978-953-51-0512-1, InTech, Available from:

http://www.intechopen.com/books/stoichiometry-and-materials-science-when-numbers-matter/study-of-solidphase-chemical-reactions-using-xps

\section{INTECH}

open science | open minds

\section{InTech Europe}

University Campus STeP Ri

Slavka Krautzeka 83/A

51000 Rijeka, Croatia

Phone: +385 (51) 770447

Fax: +385 (51) 686166

www.intechopen.com

\section{InTech China}

Unit 405, Office Block, Hotel Equatorial Shanghai

No.65, Yan An Road (West), Shanghai, 200040, China

中国上海市延安西路65号上海国际贵都大饭店办公楼 405 单元

Phone: +86-21-62489820

Fax: $+86-21-62489821$ 
(C) 2012 The Author(s). Licensee IntechOpen. This is an open access article distributed under the terms of the Creative Commons Attribution 3.0 License, which permits unrestricted use, distribution, and reproduction in any medium, provided the original work is properly cited. 